See discussions, stats, and author profiles for this publication at: https://www.researchgate.net/publication/341233096

\title{
3D phase-resolved wave modelling with a non-hydrostatic ocean circulation model
}

Preprint · May 2020

CITATIONS

0

3 authors, including:

and

Patrick Marsaleix

French National Centre for Scientific Research

127 PUBlicAtions 3,335 CITATIONS

SEE PROFILE

Some of the authors of this publication are also working on these related projects:

Project MERMEX-DEWEX View project

Project Development of unstructured WWIII View project
READS

106

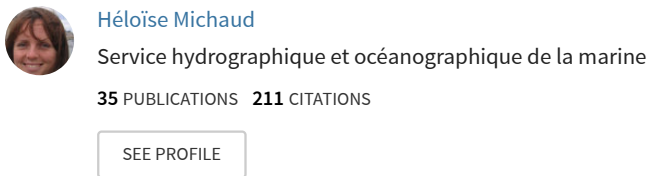


1 3D phase-resolved wave modelling with a non-hydrostatic ocean circulation model

2 Patrick Marsaleix (1), Héloïse Michaud (2), Claude Estournel (1)

3

4 (1) Laboratoire d'Aérologie, Université de Toulouse, CNRS, UPS, 14 avenue Edouard Belin, 531400 Toulouse, France

(2) Shom - Antenne de Toulouse, 42 avenue G. Coriolis, 31057 Toulouse, France.

7

8 Key words: phase-resolved wave modelling, wave breaking, rip currents, non-hydrostatic, 9 ocean circulation model.

Corresponding author: Patrick Marsaleix, patrick.marsaleix@aero.obs-mip.fr

\section{Abstract}

A phase-resolved wave model is derived from an ocean circulation model for the purpose of studying wave-current effects in nearshore zones. One challenge is to adapt the circulation model to the specificities of wave physics. This mainly concerns the consideration of nonhydrostatic effects and the parametrization of wave breaking. The non-hydrostatic pressure is calculated using the artificial compressibility method (ACM). The ACM-induced errors on wave dispersion properties are examined in detail in the context of the linear theory using idealized test cases. The possible compromise between the precision achieved on nonhydrostatic physics and the adjustable CPU cost of the ACM method is looked at in detail. The modification of the wave characteristics by the bathymetric slope and the breaking of waves are then examined from a linear slope beach laboratory experiment. Finally the model is evaluated on the issue of rip currents and their feedback on the wave field using a laboratory experiment of a beach with a bar intersected by channels. 
The interactions between waves and current play a major role in coastal modelling studies and are important to correctly reproduce the nearshore circulation. Coastal engineering applications need realistic descriptions of waves and currents, their transformations towards the nearshore and behaviours with artificial coastal structures for the design of harbours or dykes to prevent overtopping and to reduce submersion or erosion risks and this is only possible with a circulation model that reproduces explicitly the waves. Taking into account the waves in the circulation models allows on the one hand the representation of the residual flows generated by the waves such as the Stokes drift (Mellor 2003) or the rip currents (Castelle et al, 2016) and on the other hand, to take into account turbulence generation by waves in the turbulent closure scheme, which improves the oceanic surface and bottom boundary layers, the vertical current shear, and the vertical temperature and salinity profiles in the circulation model (Uchiyama et al, 2010). In shallow waters, when the waves effects near the bottom are no longer negligible, the bottom stress is significantly increased, allowing the resuspension of sand and sediments (Soulsby, 1995), the Stokes drift, the undertow or rip currents then playing an important role in their transport in the water column. The wave-current interaction is also decisive for the mean surface level set-up during storms, the modification of the surface stress related to the roughness created by the waves, the modelling of the marine submersion of the coastal continental surfaces (Bertin et al, 2012), the modification of the coastline, the evolution of the shape of the seabed (Bouharguane et al, 2010) .

At the spatial scales of the coastal circulation models (see review by Klingbeil et al, 2018), such studies are mainly done using a hydrostatic circulation model coupled with a spectral phase-averaged wave model (McWilliams et al 2004, Ardhuin et al 2008, Bennis et al, 2011, Benetazzo et al, 2013, Kumar et al, 2015, Uchiyama et al, 2017). The phaseaveraged wave model feeds the circulation model with Stokes drift, residual wave pressure, momentum and turbulence production terms related to wave erosion. In return, the circulation model gives the wave model the variations of sea surface level and surface 
in the known example of rips current. The choice of a phase-averaged wave model is largely

guided by computational time considerations as it provides mean properties of the sea state.

The spectral model can indeed be used with a horizontal resolution much lower than the wave wavelengths and therefore cover large areas with a reasonable number of grid points.

An alternative is the phase-resolved model, or deterministic model, that explicitly describes the evolution of the free surface. The SWASH models (Zijlema et al, 2011), REF/DIF (Kirby and Dalrymple, 1983), FUNWAVE (Kirby et al, 1998), BOSZ (Roeber and Cheung, 2012), NHWAVE (Ma et al, 2012) are widespread examples of this type of model, very often mentioned in the wave modelling literature. The processes resulting from the effects of bathymetry such as refraction, diffraction and reflections (Magne et al, 2005), interaction with other waves or with the background circulation, infragravity waves (Bertin et al, 2018), the asymmetrical wave transformation at the approach of the breaking phase, are, in principle, better represented by this type of model (Rusu and Soares, 2013). The phaseresolved model is also well suited for sand mobilization, which is directly dependent on orbital velocity near the bottom (Bouharguane et al, 2010). The phase-resolved model has a continuous approach of frequencies and directions, while the phase-averaged model solves its equations on a discrete spectrum of frequencies and directions which results in a relative inaccuracy related to frequency and direction resolution. For example, in Michaud et al 2012, the Wavewatch III $r$ model (Tolman et al., 2016) is used with 36 discrete values of the direction leading to a truncation of the direction of the waves of the order of $10^{\circ}$. However, the phase-resolved models require finer temporal and spatial discretisation, thus larger computational resources. The resolution of the horizontal grid imposes a limit on the permissible wavelengths, truncating the shortest periods of the wave spectrum.

Deterministic nearshore models should have accurate dispersion and non-linear properties and thus should be governed by the Navier-Stokes equations (Raoult et al., 2016), using either an Eulerian approach (e.g., OpenFOAM®, Zhou et al., 2017) or a Lagrangian approach (e.g. the meshless model SPH model, Oger, 2006). These models are appropriate for local studies, although eventually limited by spurious diffusion or prohibitive 
computational time (Benoit et al., 2017). Simplifications can be made to overcome these problems: for instance, REF/DIF based on the mild slope equation (Berkhoff, 1972) neglects some nonlinear effects; SWASH can be used with depth-averaged equations with appropriate assumptions to introduce the non-hydrostatic pressure (Zijlema et al. ,2011); Boussinesq, Serre (1953) or Green-Naghdi (Green and Naghdi, 1976) models take partially into account non linear or dispersive effects (e.g., Bonneton et al.,2011a, 2011b, Tissier et al., 2012).

Within the limits of the above simplifications, phase-resolved models should theoretically be capable of simulating the low-frequency 3D processes usually modelled by circulation models, since their equations are basically the same, except for the hydrostatic assumption generally made in circulation models (Klingbeil et al. , 2018). As a result, the phase-resolved wave model is able to generate residual currents such as rip currents and therefore does not require coupling with a circulation model to simulate the coupling between waves and low frequency fields. However, it requires a significant horizontal and vertical resolution (greater than the wavelength of the waves) which limits the size of the modelled domain. To date and to our knowledge, most of the applications of the phase-resolved models seem to concern domains whose horizontal dimensions are of the order of a few kilometres in each horizontal direction (Yoon, 2014). The constant rise of computers capability suggests that phase-resolved models could now be used on larger domains covering a significant part of the continental shelf. Meanwhile, ocean circulation models currently used at coastal scales could be adapted to deterministic wave modelling with, (among other objectives), the goal of linking waves and continental shelf circulations like wind-induced eddies (Petrenko et al. 2008), or to better understand momentum and turbulence transfer in the surface layer (Deigaard and Nielsen, 2018). This assumes, however, that their equations are suitable for phase-resolved wave modelling. One of the essential points is to be able to use these models in non-hydrostatic mode. A number of articles deal with this question, for the MITgcm model (Marshall et al., 1997), POM (Kanarska and Maderich, 2003), ROMS (Kanarska et al, 2007), GETM (Klingbeil and 
113 Burchard, 2013), SYMPHONIE (Auclair et al, 2011). However, at the resolutions where

114 these models are most often used, the consideration of non-hydrostatic effects may not be

115 an essential issue (McKiver et al, 2016). The applications envisaged so far concern more the

116 propagation of internal gravity waves (Bordois et al 2017) or the convective processes

117 (Paluszkiewicz et al, 1994) than the high frequency surface waves. One of the obstacles to

118 the deterministic modelling of waves over large domains is the cost of the non-hydrostatic

119 solver (Klingbeil et al, 2018). As previously mentioned, in the field of phase-resolved wave

120 modelling, the common alternative to this difficulty is to use simplified models adapted to the

121 physical specificities of waves (Bonneton et al.,2011a, 2011b). In the field of ocean

122 circulation modelling, the most widespread approach to the non-hydrostatic problem, on the

123 contrary, is to take into account the whole complexity of the problem. It leads concretely to

124 the resolution of a Poisson equation for the non-hydrostatic pressure. This is a global approach insofar as this leads to a system of linear equations making all the points of the numerical domain interdependent. Its resolution is in principle costly and currently constitutes a challenge in the perspective of large numerical domains of several thousand points in each direction (Roulet et al., 2017). The sigma coordinate widely used in coastal modelling also increases the cost of the resolution for two main reasons. The first reason is that the time variation of the position of the vertical levels (related to the movement of the free surface) forces to update regularly the coefficients of the main matrix of the system and its possible preconditioning. The possible representation of the wetting-drying zones adds a similar difficulty. The second reason is that the sigma coordinate transformation increases

134 the number of nonzero coefficients of the principal matrix. Roulet et al. 2017 propose to reduce this number by reformulating the momentum equations. The same authors also evoke the perspective of the multigrid approach to significantly reduce the cost of calculation. An application in the vertical direction is proposed by Shi et al. (2015).

The alternative to the global approach is the local approach based on the direct resolution of non-hydrostatic pressure. Various methods have been proposed so far (Johns 140 1991, Klingbeil and Burchard, 2013, Bordois et al, 2017, Lee et al, 2006). Among them, Lee 
141 et al, 2006 (hereafter L06) ACM (artificial compressibility method) method has a reasonable

142 numerical cost and its implementation in a model is easy. A method derived from L06 is 143 implemented in the SYMPHONIE circulation model (Marsaleix et al, 2008) and tested in the 144 aforementioned context, namely the high frequency surface waves and the associated 145 residual circulations. The purpose of the present paper is to assess this model on the 146 principal issues of wave modelling. This concerns on the one hand the dispersion properties,

147 the vertical shear of the current, the asymmetrical wave transformation at the approach of 148 the breaking conditions, the decay of the waves in the breaking zone. On the other hand, 149 this concerns the residual waves-related circulation such as the stokes transport, the 150 alongshore coastal drift, the mean sea level, rip currents and their feedback on the waves. 151 The article is organized as follows: chapter 2 deals with the implementation of the ACM 152 method in the ocean circulation model, chapter 3 gives an analytical solution verifying the 153 ACM equations for low amplitude waves, chapter 4 describes academic and laboratory test 154 cases and finally chapter 5 provides a summary and the conclusions.

155

1562 Model description

157

158

\subsection{Equations}

159

160 For the sake of clarity the problem is first presented in a simplified way, in a vertical 2D plane 161 and presenting only the terms that dominate wave physics:

162

$163 \quad \frac{\partial u}{\partial t}=-g \frac{\partial \eta}{\partial x}-\frac{\partial q}{\partial x}$

164

$165 \frac{\partial w}{\partial t}=-\frac{\partial q}{\partial z}$

166

167 
where $q$ is the non-hydrostatic pressure times the water density, $u$ and $w$ the horizontal and vertical current, $\eta$ the sea surface height. As in L06 the hypothesis of incompressibility is retained to deduce the surface elevation:

$172 \quad \frac{\partial \eta}{\partial t}=-\frac{\partial}{\partial x} \int_{-h}^{\eta} u d z$

173

174 Equation (2) is in practice not calculated by the model. Instead, the combination of (1) and

175 (2) that leads to the Poisson equation is used:

$177 \quad \frac{\partial}{\partial t}\left(\frac{\partial u}{\partial x}+\frac{\partial v}{\partial y}+\frac{\partial w}{\partial z}\right)=-g \frac{\partial^{2} \eta}{\partial x^{2}}-\frac{\partial^{2} q}{\partial x^{2}}-\frac{\partial^{2} q}{\partial z^{2}}$

179 The left-hand side of the Poisson equation is ideally zero in the case of the incompressible 180 hypothesis. Without questioning this hypothesis of incompressibility, the particularity of the 181 L06 method is however to allow the left-hand side of (4) to be small and not to equal zero. Before detailing the approach of L06, some comments can be made on the possibility of not cancelling strictly the left-hand side of (4). It can be noted, for example, that non-hydrostatic incompressible models can use iterative solvers whose convergence is de facto imperfect, precluding a strict cancellation of the left-hand side of (4). This approximation is currently accepted because the physical coherence is preserved if the convergence of the solver is sufficiently precise. We can also recall that a model such as the one used in this study does not formally represent (4) but its discrete approximation in the finite difference sense. Not strictly cancelling the left-hand side of (4) induces an error whose order of magnitude can be compared to the discretization errors of the other terms. For example, given the numerical scheme used here, the error made on the horizontal laplacian of $q$ at the right-hand side of (4) is of the order of (APPENDIX A):

193

$194 \quad \frac{d x^{2}}{12} \frac{\partial^{4} q}{\partial x^{4}} \approx \frac{d x^{2}}{12} k^{4} q$ 
196 where $k$ is the wavenumber. This discretization error makes it possible to consider a possible 197 error on the left-hand side, thus relativizing the question to the transformation of the left-hand 198 side of (4) into a term of artificial compressibility as described by L06 (see equation 2 in 199 L06). In practice, the ACM method of L06 leads to the reformulation of (4) as follows:

200

$201 \quad \frac{1}{\alpha^{2}} \frac{\partial^{2} q}{\partial t^{2}}=g \frac{\partial^{2} \eta}{\partial x^{2}}+\frac{\partial^{2} q}{\partial x^{2}}+\frac{\partial^{2} q}{\partial z^{2}}$

202

203 Assuming a sinusoidal form for $q$, the artificial compressibility term at the left-hand side of 204 (6) is of the same order as $\left(\frac{\omega}{\alpha}\right)^{2} q=\left(\frac{c}{\alpha}\right)^{2} k^{2} q \quad(c$ is the celerity of the waves), which, under 205 certain conditions, can become of an order of magnitude comparable to (5) (ie $\left(\frac{c}{\alpha}\right)^{2}$ 206 comparable to $\frac{d x^{2}}{12} k^{2}$ ), insofar as it is recommended to use the ACM method with $\alpha$ such that $207\left(\frac{c}{\alpha}\right)^{2}<<1$. This point is closely examined later in the article.

In fact, ideally, in compressible theory, the $\alpha$ constant should be the speed of the 209 acoustic waves, actually much higher than $c$. However, as pointed out by Mahadevan et al 210 (1996), this would have the consequence of requiring an extremely small time step, highly 211 increasing the computational cost. This type of approach therefore generally uses a much 212 lower $\alpha$ value in order to increase the time step and thus makes the cost of the calculation 213 affordable. It is therefore noted that the true compressibility of the ocean is not respected by 214 this approach which relies more on numerical considerations than on a real physical 215 justification (resulting in the expression "artificial compressibility" used by L06). On the other hand, apart from the equation (6), the other equations of the model strictly respect the

217 incompressibility framework. Although greatly diminished by the computational time 218 constraint just mentioned, the value of $\alpha$ must nevertheless remain sufficiently large to allow 219 rapid adjustment of equation (6) to variations in the hydrostatic forcing terms. This property 
is essential for the accuracy of the non-hydrostatic behavior of the model. In practice, $\alpha$ must

221 remain larger than the phase speed of gravity waves (Bordois et al, 2017).

222 The resolution of the Poisson iterative equation (6) can be done with the same time step as 223 the other equations. In practice its determination is done from the stability criterion

224

225

226

227

228

229

230

231

232

233

234

235

236

237

238

239

240

$\frac{1}{\alpha^{2}} \frac{\partial^{2} q}{\partial t^{2}}=-\frac{\partial}{\partial x}\left(\frac{\partial u}{\partial t}\right)^{*}-\frac{\partial}{\partial y}\left(\frac{\partial v}{\partial t}\right)^{*}+\frac{\partial^{2} q}{\partial x^{2}}+\frac{\partial^{2} q}{\partial y^{2}}+\frac{\partial^{2} q}{\partial z^{2}}$

241

242 Where $\left(\frac{\partial u}{\partial t}\right)^{*}$ and $\left(\frac{\partial v}{\partial t}\right)^{*}$ represent hydrostatic terms (in practice all the terms of the right-

243 hand side of (7) and (8) except $r \frac{\partial q}{\partial x}$ and $\left.r \frac{\partial q}{\partial y}\right)$. At this stage, $r$ at the right-hand side of (7)

\subsection{Implementation in the SYMPHONIE model}

The model used is the ocean circulation model SYMPHONIE (Marsaleix et al. 2008). As we focus on surface waves, the effect of temperature and salinity is neglected. For the sake of clarity the equations are given in a Cartesian coordinate system but the model actually uses a system of generalized sigma coordinates. The momentum equations are given by:

$\frac{\partial u}{\partial t}=-\frac{\partial u u}{\partial x}-\frac{\partial v u}{\partial y}-\frac{\partial w u}{\partial z}+f v-g \frac{\partial \eta}{\partial x}-r \frac{\partial q}{\partial x}+\frac{\partial}{\partial z}\left(K_{m} \frac{\partial u}{\partial z}\right)+\tau_{u}$

and (8) is equal to one but we will see later that a value other than 1 can be used to improve 
245 the accuracy of the dispersion relation and currents of short waves. The incompressibility

246 hypothesis gives the vertical velocity and elevation of the surface:

247

$248 \quad \frac{\partial u}{\partial x}+\frac{\partial v}{\partial y}+\frac{\partial w}{\partial z}=0$

$249 \quad \frac{\partial \eta}{\partial t}=-\frac{\partial}{\partial x} \int_{-h}^{\eta} u d z-\frac{\partial}{\partial y} \int_{-h}^{\eta} v d z$

250

251 The vertical diffusion coefficient is calculated by the k-epsilon scheme (Rodi, 1987) and

$252\left(\tau_{u}, \tau_{v}\right)$ are breaking terms parameterized as a horizontal diffusion:

253

$254 \quad \tau_{u}=\frac{\partial}{\partial x}\left(v_{b} \frac{\partial u}{\partial x}\right)+\frac{1}{2} \frac{\partial}{\partial y}\left(v_{b}\left(\frac{\partial u}{\partial y}+\frac{\partial v}{\partial x}\right)\right)$

$255 \quad \tau_{v}=\frac{\partial}{\partial y}\left(v_{b} \frac{\partial v}{\partial y}\right)+\frac{1}{2} \frac{\partial}{\partial x}\left(v_{b}\left(\frac{\partial u}{\partial y}+\frac{\partial v}{\partial x}\right)\right)$

256

257 The eddy viscosity of breaking $v_{b}$ is calculated from a breaking criterion which will be 258 discussed in a section to follow. Bottom and surface boundary conditions are as follows: at 259 the surface $q=0, \frac{\partial(u, v)}{\partial z}=0$ and at the bottom $\frac{\partial q}{\partial z}=0, \rho_{0} K_{m} \frac{\partial(u, v)}{\partial z}=\left(\tau_{b}^{x}, \tau_{b}^{y}\right)$ where $\rho_{0}$ is the 260 sea water density and $\left(\tau_{b}^{x}, \tau_{b}^{y}\right)$ are the components of the bottom stress. The bottom stress 261 is parameterized as in Blumberg and Mellor (1987), from a quadratic relationship of the bottom current and a drag coefficient derived from a roughness length.

The equations are calculated with a forward-backward time scheme detailed in APPENDIX B. Considering that short surface waves are intrinsically three-dimensional, the barotropic-baroclinic time splitting commonly used in circulation models for calculating separately barotropic and baroclinic velocities (Blumberg and Mellor 1987) can not applied here. In practice, all the equations are calculated with the same time step. 
272 backward scheme. In the case of the hydrostatic model, the criterion of stability with respect 273 to surface waves would be:

274

$275 d t_{\max }=\frac{d x}{\sqrt{2}} \frac{1}{\sqrt{g h_{\max }}}$

276

277 where $d x$ is the horizontal resolution, $h_{\max }$ is the maximum depth of the domain and

278 therefore $\sqrt{g h_{\max }}$ is the maximum theoretical phase speed expected for surface waves. In 279 the non-hydrostatic model, the calculation of the equation for $q$ imposes a more drastic 280 additional criterion since it depends on $\alpha$ that is greater than $\sqrt{g h_{\max }}$. It is not trivial to find a 281 simple expression similar to (14) but, unsurprisingly, it appears in simulations performed by 282 the authors that the maximum value of the time step allowed by the non-hydrostatic model 283 was close to $\frac{d x}{\sqrt{2}} \frac{1}{\alpha}$, ie (14) with $\sqrt{g h_{\max }}$ replaced by $\alpha$. In practice, in all the simulations 284 presented, the time step of the non hydrostatic model is given by a fraction $(70 \%)$ of this 285 criterion to include other stability criteria than gravity waves such as those resulting from 286 non-linearities, namely:

287

$288 d t=0.7 \frac{d x}{\sqrt{2}} \frac{1}{\alpha}$

289

290 The determination of $\alpha$ is therefore related to the determination of the time step. The larger 291 the first, the smaller is the second. The quantity $N$ which represents the ratio of the 292 theoretical time step of the hydrostatic case to the time step of the non-hydrostatic case, is 293 introduced: 
297 which, using (14) and (15), is equivalent to

298

$299 \quad N=\frac{1}{0.7} \frac{\alpha}{\sqrt{g h_{\max }}}$

300

$301 N$ is a key parameter of the problem. The larger it is, the smaller is the model time step and 302 the better is the accuracy expected on the non-hydrostatic term. Since $d t_{\max }$, is the time 303 step that would normally have the hydrostatic model in similar conditions, the ratio $N$ is a good indicator of the relative additional cost associated with the non hydrostatic effect. The evaluation of this additional cost must also take into account that an additional equation (for q) has been added to the equations of the hydrostatic model. The latter essentially counts 4 three-dimensional equations, two for the horizontal components of the current, one for the turbulent kinetic energy and one for its dissipation rate. The relative numerical excess cost of

309 the equation for $q$ is therefore of the order of $N \frac{4+1}{4}=1.25 \mathrm{~N}$.

310

311

\section{Analytical solutions}

312

313 Analytical solutions verifying model equations in the context of linear theory are now

314 presented. We consider a 2D vertical plan, a linearized version of (7),

315

$316 \quad \frac{\partial u}{\partial t}=-g \frac{\partial \eta}{\partial x}-r \frac{\partial q}{\partial x}$

317

318 the continuity equation,

319

$320 \frac{\partial u}{\partial x}+\frac{\partial w}{\partial z}=0$ 
and the non-hydrostatic pressure equation (6). The elevation of the surface is of the form $323 \eta=\eta_{0} e^{i(k x-\omega t)}$. The non-hydrostatic pressure satisfying equation (6) is of the form:

324

325

$q=-\left(g / \mu^{2}\right) \eta+2 P^{0} e^{i(k x-\omega t)} \cosh (\mu k(z+h))$

326

327 with

328

$329 \quad \mu^{2}=\left(1-\frac{\omega^{2}}{k^{2} \alpha^{2}}\right)$

330

331

Where $\alpha$ appears in the left hand side of (6) and in the definition of $N$ calculated by (17).

332

Using (20) and the surface condition $q=0$ leads to:

333

334

$q=\frac{g}{\mu^{2}} \eta(-1+\cosh (\mu k(z+h)) / \cosh (\mu k h))$

335

336 Using (22) and (18) gives the horizontal current:

337

338

$u=g \frac{k}{\omega} \eta\left[1+\frac{r}{\mu^{2}}\left(\frac{\cosh (\mu k(z+h))}{\cosh (\mu k h)}-1\right)\right]$

339

340 Using (23) and the continuity equation (19) to derive the vertical velocity, then applying the

341 surface condition $w=\frac{\partial \eta}{\partial t}$, leads to the dispersion relation:

342

$343 \quad \omega^{2}=k^{2} g\left(1-\frac{r}{\mu^{2}}\right) h+\frac{r}{\mu^{3}} g k \tanh (\mu k h)$

344

345 Note that if $r=1$ and if $N$ (therefore $\alpha$ ) tends to infinity (leading to the cancellation of the left-

346 hand side of $(6)$ and $\mu=1$ ) the dispersion relation (24) becomes equivalent to the usual

347 dispersion relation (Kinsman, 1965): 
$349 \omega^{2}=g k \tanh (k h)$

350

351 while, using (23) and (25), we find the usual solution for the horizontal current (Kinsman, 352 1965):

353

$354 u=\omega \eta \frac{\cosh (k(z+h))}{\sinh (k h)}$

355

356

This result shows that the ACM method becomes equivalent to the classical non-

357 hydrostatic incompressible method if $N$ is sufficiently large. In the following, we distinguish

358 the dispersion relations of the incompressible non-hydrostatic linear theory (ie (25)), of the 359 non-hydrostatic ACM theory (ie (24)) and of the hydrostatic theory (ie (27)):

360

$361 \omega / k=\sqrt{g h}$ 

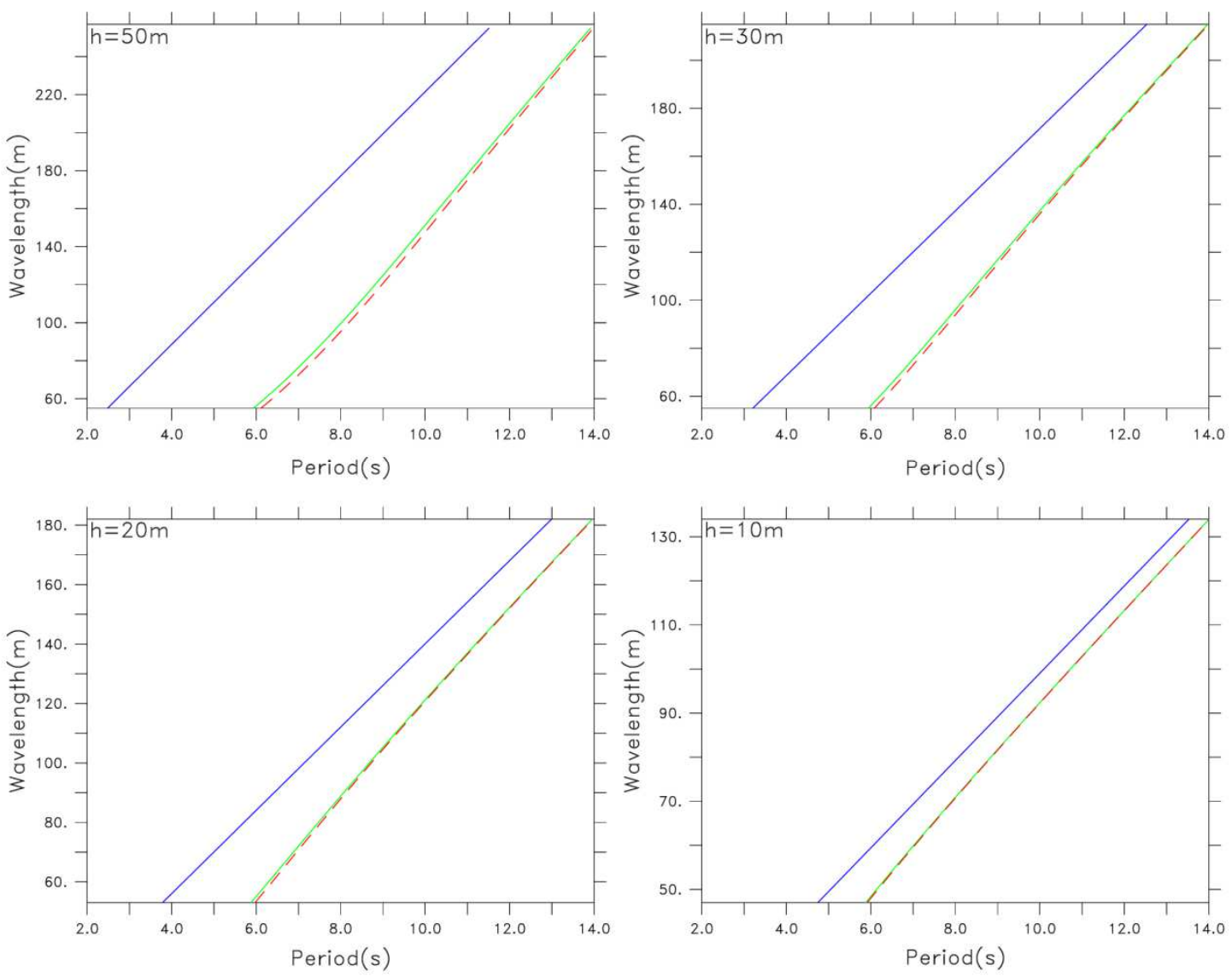

362

363 Figure 1. Dispersion relations at different bottom depth. Blue line: hydrostatic linear theory (27).

364 Green line: incompressible non-hydrostatic theory (25). Dashed red line: ACM theory (24) with $r=1$ and $N=5$.

Figure 1 shows these different dispersion relations for periods ranging from $6 \mathrm{~s}$ to $14 \mathrm{~s}$

368 (this choice will be discussed in the next section), for different bathymetry values and $N=5$.

369 The ACM theory with $r=1$ provides a considerable improvement over the hydrostatic theory

370 but overestimates the non-hydrostatic effect (Figure 1: the dashed red curve is always below

371 the green curve). This overestimation increases with bathymetry. At shallow depths the agreement is very satisfactory, but the non-hydrostatic effect is also less significant (Figure $1, h=10 m)$. 
376 hydrostatic incompressible theory. Frequencies produced by (24) and (25) are actually 377 equivalent if

378

$379 \quad r=\mu^{3} \frac{\tanh (k h)-k h}{\tanh (\mu k h)-\mu k h}$

380

381 Horizontal currents (23) and (26) are identical on the surface without condition on $r$ and $\mu$,

382 while near the bottom they are equivalent if

383

$384 r=\mu^{2}\left(\frac{1}{\cosh (k h)}-1\right)\left(\frac{1}{\cosh (\mu k h)}-1\right)^{-1}$

385
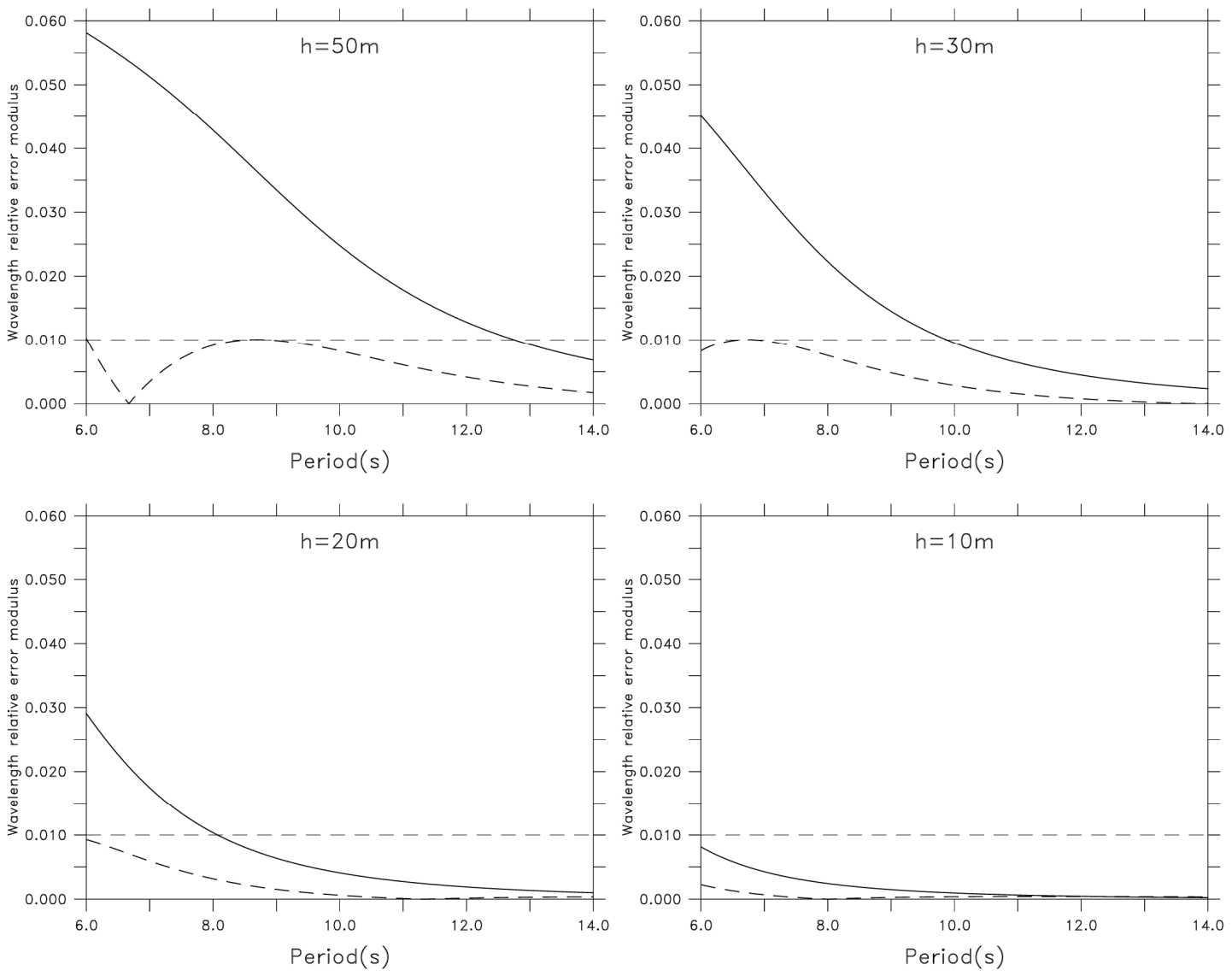
Figure 2. Relative error on the wavelength computed by the ACM dispersion relation (24) at different

388 bottom depth and $N=5$. Solid line: $r=1$. Dashed line $r=0.9845$. The horizontal dashed line indicates 389 an error level of $1 \%$.

390

391 Expressions (28) and (29) suggest the possibility of optimizing $r$ according to the 392 local values of the depth and the dominant period of waves. This point will be addressed in 393 future applications. At this early stage of our study, $r$ will be considered constant. Figure 2 394 shows the absolute value of the relative error on the wavelength, $\left|L_{A C M}-L\right| / L$, as a function 395 of the period, $L_{A C M}$ being deduced from (24) and $L$, the "true" wavelength, from (25). 396 Relative errors are more important for small periods and for large bathymetry values. Errors 397 of the order of several \% are expectable with $r=1$. On the other hand, it is possible to 398 reduce the error level below $1 \%$, for all the values of period and bathymetry considered in 399 Figure 2, with $r=0.9845$.

$400 \quad$ The fact that (28) and (29) are not identical shows that it is not possible to strictly 401 cancel the error on the current and on the dispersion relation with the same value of $r$. 402 However as shown in Figure 3 for $h=50 m$ and a period of $10 \mathrm{~s},(28)$ and (29) give very similar 403 values of $r$ so that it is possible to find a value of $r$ that reduces both the current and 404 dispersion relation errors (this will be discussed in the next section). Finally we note that a 405 compromise on these two possible values of $r$ is all the easier to find if $N$ is large because 406 (28) and (29) converge both to $r=1$. 


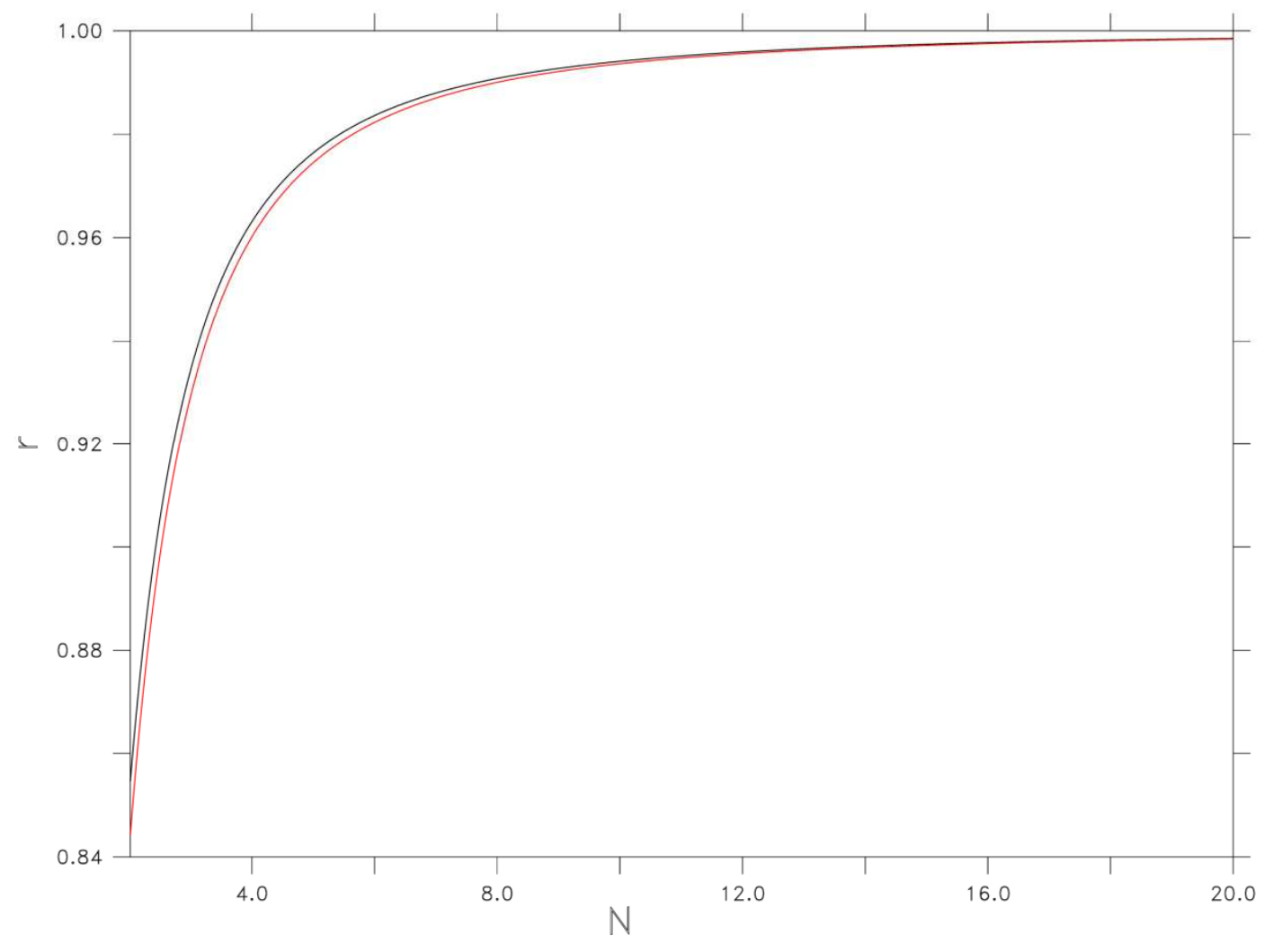

407

408 Figure 3: $r$ computed with (28) (Black line) and (29) (red line) as a function of $N$ for $h=50 m$ and a 409 period of 10 s.

410

411 In conclusion, the analytical solutions corresponding to the linearized equations of the ACM 412 method show a satisfactory representation of the non-hydrostatic effect in the range of 413 periods concerned by the future applications of the model to the near shore zone. The 414 accuracy however depends on the setting of parameters such as $N$ and $r$. Since the 415 accuracy of a numerical model also depends on many other factors related to the 416 discretization of equations, such as spatio-temporal resolution and relevance of numerical 417 schemes, the above theoretical analysis is now completed with numerical experiments. 


\subsection{Wave propagation in the context of linear theory}

422

423

In this section, we check the ability of the model to correctly represent the physical

characteristics of the simulated wave thanks to a series of test cases consisting in exploring the dispersion properties of the waves. We consider here low amplitude waves $(1 \mathrm{~mm})$ in order to be within the framework of the linear approximation for which simple analytical solutions exist (Kinsman, 1965). The objective is to define the values of $N$ which lead to a reasonable compromise between the computation cost (consideration encouraging to lower $N)$ and the precision of the dispersion properties (consideration encouraging to increase $N$ ). We use a domain with a flat bottom at $50 \mathrm{~m}$ depth, a periodic condition in the direction normal to wave propagation. The horizontal resolution is $5 \mathrm{~m}$ and 20 vertical levels are used. The waves are generated at the entrance of the domain, while the fields are at rest at the initial state. The opposite boundary is closed but the dimension in the direction of propagation is large enough, so that the waves do not have time to reflect on it before the end of the simulation (10 minutes). This first numerical experiment is carried out with $r=1$. recommended by Blayo and Debreu (2005), an outgoing field is defined as the difference between the total field and the incoming field. We thus define anomalies of currents, sea surface height and non-hydrostatic pressure, $u^{\prime}=u-u_{w}, \eta^{\prime}=\eta-\eta_{w}, q^{\prime}=q-q_{w}$ where $u_{w}, \eta_{w}, q_{w}$ are prescribed by the analytical solution of the short wave linear theory. We then

441 assume that the incoming short waves do not reflect at the coast because of dissipation and 442 that waves propagating offshore are at lower frequencies (they may consist of infra-gravity 443 waves if the conditions of their generations are met (Bertin et al, 2018)). The radiative 444 conditions, $u^{\prime}= \pm g \eta^{\prime} / c$ and $\partial q^{\prime} / \partial t \pm c \partial q^{\prime} / \partial x=0$, are therefore applied using the long 445 wave phase speed $c=\sqrt{g h}$. 
447 compare it with that predicted by the theoretical relation dispersion of the incompressible 448 linear theory (25). The modelled wavelength is diagnosed from the distance separating two 449 successive identical values of the surface level in the direction of propagation. The grid 450 points chosen for the calculation of this diagnosis are located beyond a distance of $625 \mathrm{~m}$ 451 from the open boundary and, moreover, the diagnosis is only calculated once the transient 452 regime is reached (i.e. once the wave front has passed these points). A time average of the 453 values obtained is finally calculated. A set of simulations is done to estimate the precision on 454 (25) as a function of $N$ (ranging from 2 to 20) and of the wave period. The smallest period 455 considered here is 6 seconds, associated with a wavelength of $56 \mathrm{~m}$ for $h=50 \mathrm{~m}$, represented 456 by 11 grid points given the horizontal resolution of $5 \mathrm{~m}$. This minimum value is considered as 457 a reasonable threshold with respect to the capacity of the C-grid to represent the small 458 wavelengths. The maximum period considered is $14 \mathrm{~s}$ which is associated with a wavelength 459 of approximately $250 \mathrm{~m}$. Periods greater than $14 \mathrm{~s}$ are not considered because their 460 wavelengths, much larger than the depths envisaged, render them slightly non-hydrostatic 461 and therefore not interesting from the point of view of the authors. 

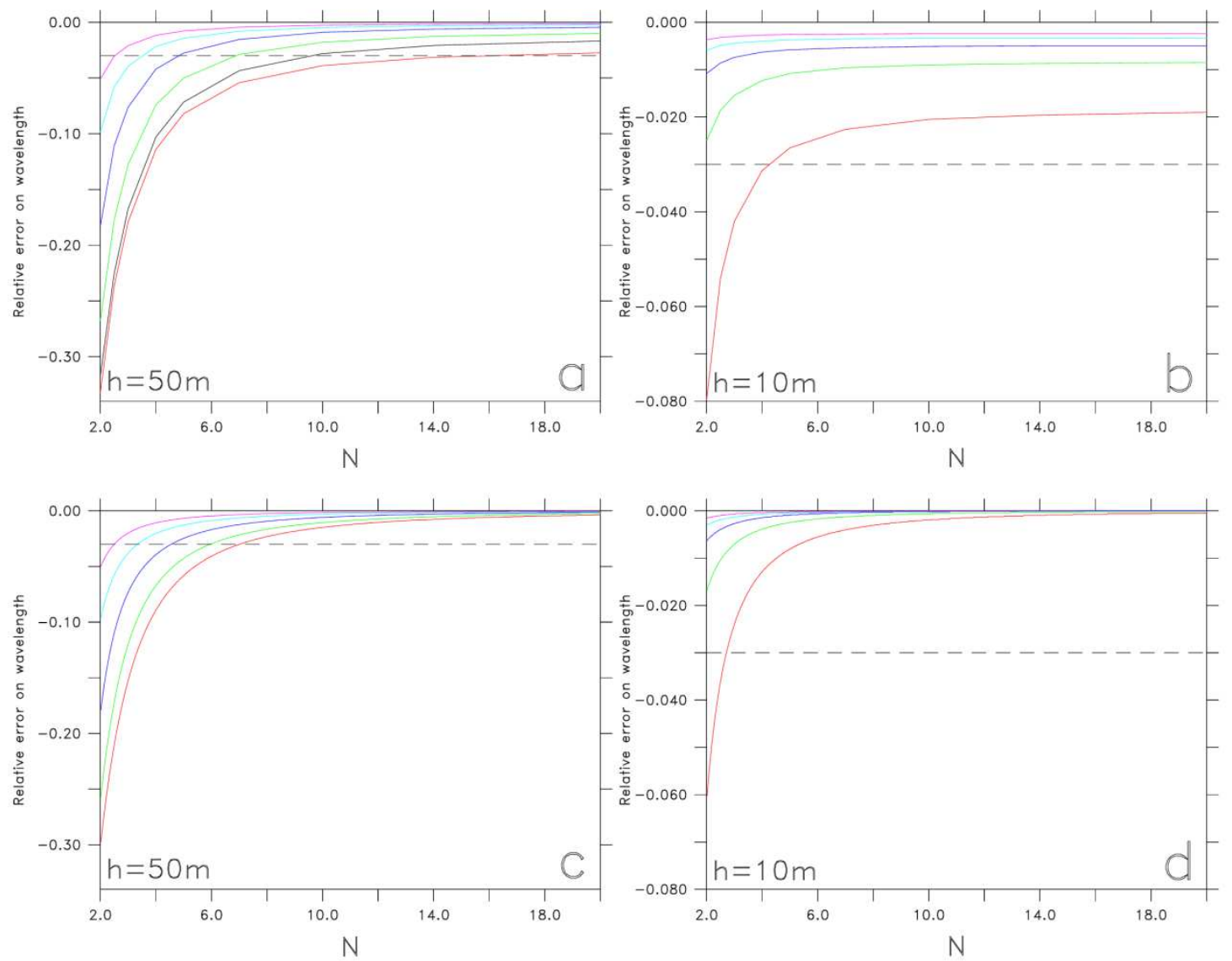

462

463 Figure 4: Relative error on the wavelength $\left(L^{*}-L\right) / L$ where $L$ is given by the incompressible linear 464 theory and $L^{*}$ is either deduced from simulations with 20 vertical levels and $d x=5 m$ (figures $a$ and $b$ ) or from the ACM linear theory (figures $c$ and $d$ ). The horizontal dashed line indicates a relative error of -3\%. Each curve corresponds to a period: $14 s$ (magenta), 12s (cyan), 10s (blue), 8s (green), $6 \mathrm{~s}$ (red). The black curve (figure a) corresponds to a period of $6 s$ and $d x=2.5 m . h=50 m$ (figures $a$ and c), or $h=10 m$ (figures $b$ and $d$ )

471 Figure 4 shows the relative error on the wavelength, $\left(L^{*}-L\right) / L$, where the "true" wavelength $472 L$ is given by the incompressible linear theory (25) and $L^{*}$ is either deduced from the model or from the ACM linear theory (24) with $r=1$. Errors decrease when $N$ increases. As expected 474 from the previous section, the errors are stronger when the bottom depth is important. This 
could be an issue near the model open boundaries where the most important depths are 476 normally expected. To our knowledge, near shore modelling studies mostly focus on areas 477 where bathymetry does not exceed a few tens of meters. We thus assume that $50 \mathrm{~m}$ could be a plausible upper limit for realistic models of the near shore zone (at least for the time being). In the following, the case $h=50 \mathrm{~m}$ is the subject of greater attention.

The model errors are generally similar to those predicted by the ACM linear theory

481

482

483

484

485

486

487

488

489

490

491

492

493

494

495 (Figure 4a and Figure 4c). A notable difference is that errors tend to zero when $N$ increases in the case of the theory but not in the case of the model. This is explained by additional sources of error, of numerical origin, in the case of the model. These numerical errors are more important for small periods. For example, for a period of $6 \mathrm{~s}$, the residual error for large values of $N$ is around $3 \%$ for $h=50 \mathrm{~m}$ and around $2 \%$ for $h=10 \mathrm{~m}$ (Figure $4 \mathrm{a}$ and Figure 4b). The agreement between the model and the ACM linear theory is less good for $h=10 \mathrm{~m}$, especially for a period of $6 s$ (Figure $4 b$ and Figure $4 d$ ). The relative predominance of numerical errors is here related to the decrease of the wavelength caused by the decrease of $h$. For a period of $6 \mathrm{~s}$ and $h=10 \mathrm{~m}$, the wavelength is indeed around $48 \mathrm{~m}$, i.e. less than 10 grid points while the wavelength is around $56 \mathrm{~m}$ i.e. more than 11 points when $h=50 \mathrm{~m}$. Actually, when $h$ decreases, causing the wavelength to decrease, the resolution of the horizontal grid eventually becomes insufficient to represent the shortest waves, unless wave breaking occurs before this critical situation.

Model errors are also sensitive to vertical resolution. The accuracy of the vertical profile of $q$ and current depends on the number of vertical levels. The shortest wavelengths are particularly sensitive to this error because the vertical profile of $q$ is more non-linear and is established on a smaller depth. Figure 5 shows that for the 6 s period, the relative error is much smaller with 80 levels than with 10 or 20 . Using more vertical levels reduces the residual error (but does not completely eliminate it). The error levels obtained with 40 levels or 80 levels are however equivalent. For larger periods (results not shown), the sensitivity of the error to the vertical resolution is smaller, plausibly because the vertical profile of the fields, whose characteristic scale of decay is related to the wavelength, is less sheared. 


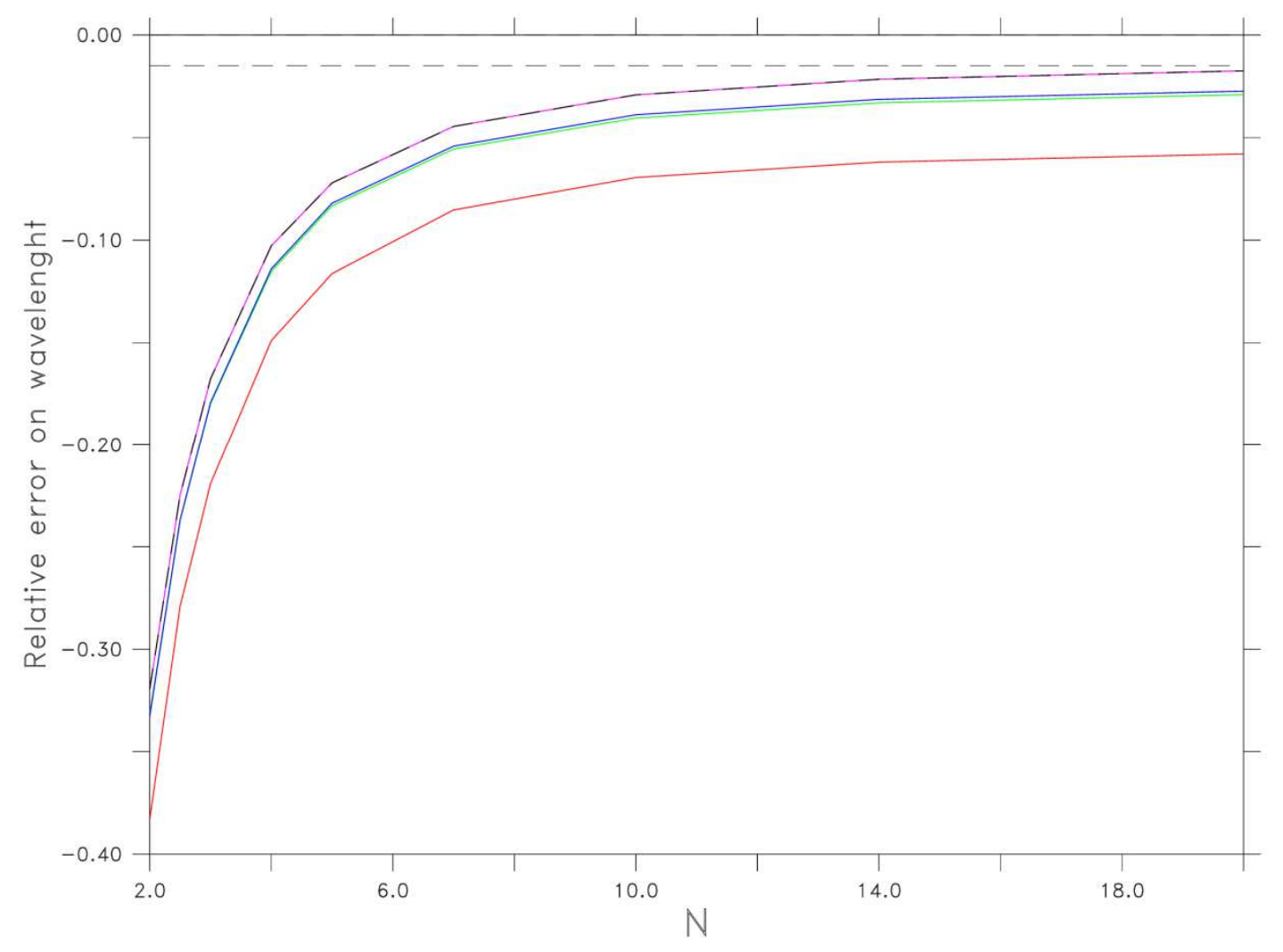

503

504 Figure 5: relative error on the wavelength (vertical axis) as a function of $N$ (horizontal axis). $h=50 \mathrm{~m}$.

$505 d x=5 m$. The horizontal dashed black line indicates a relative error of $-1.5 \%$. The period is 6 s. Each

506 curve corresponds to a different number of vertical levels. From top to bottom: 80 regular levels

507 (magenta), 40 regular levels (dashed bold black line), 20 regular levels (blue), 10 levels tightened

508 near the surface (green), 10 regular levels (red).

509

510 The "minimum" error level, around $1.5 \%$ in Figure 5 (case 80 levels, $N=20$ ), is attributable to

511 the time-stepping method itself and no longer depends on the non-hydrostatic aspects. This

512 aspect of the error can be examined in the hydrostatic model by re-invoking the simple

513 assumptions we made in section 2.1. Combining the equation (1) (now without term $q$ ) and

514 (3) the surface elevation is given by: 
$516 \quad \frac{\partial^{2} \eta}{\partial t^{2}}=c^{2} \frac{\partial^{2} \eta}{\partial x^{2}}$

517

518 Given the numerical schemes used by our model, the error is respectively of the order of

$519 \frac{d t^{2}}{12} \frac{\partial^{4} \eta}{\partial t^{4}} \approx \frac{d t^{2}}{12} \omega^{4} \eta$ and $c^{2} \frac{d x^{2}}{12} \frac{\partial^{4} \eta}{\partial x^{4}} \approx c^{2} \frac{d x^{2}}{12} k^{4} \eta$ for the left- and right-hand sides of (30). These

520 errors are estimated from the first terms of a Taylor expansion (APPENDIX C). The

521 compensation of temporal and spatial discretization errors is a characteristic of this type of

522 discrete equation (Lemarié et al, 2015, page 127 paragraph 2). Here we see that the two

523 above errors are compensated when the time step is at its theoretical maximum, i.e.

$524 d t=d x / c$ (with $c=\omega / k$ ). When the time step is significantly smaller (consequence of the

525 increase of $N$ ) the time-dependent error becomes negligible and the error is reduced to the

526 error of the right-hand side, $c^{2} \frac{d x^{2}}{12} \frac{\partial^{4} \eta}{\partial x^{4}}$, also equivalent to $-c^{2} \frac{d x^{2}}{12} k^{2} \frac{\partial^{2} \eta}{\partial x^{2}}$, and then the

527 discretization of the (30) equation tends to behave like (APPENDIX C):

528

$529 \quad \frac{\partial^{2} \eta}{\partial t^{2}} \approx c^{2} \frac{\partial^{2} \eta}{\partial x^{2}}-c^{2} \frac{d x^{2}}{12} k^{2} \frac{\partial^{2} \eta}{\partial x^{2}}+\cdots$

530

531 so that the modelled phase speed approaches $c \sqrt{1-\frac{d x^{2}}{12} k^{2}} \approx c\left(1-\frac{d x^{2}}{24} k^{2}\right)$. The relative

532 error on the phase speed is therefore of the order of $\frac{d x^{2}}{24} k^{2}=\frac{(2 \pi / n)^{2}}{24}$ where $n$ is the number

533 of grid points per wavelength. For the shortest wavelengths considered (for which $n$ is

534 around 10), the relative error on $c$ is therefore of the order of $1.5 \%$. Given that the period is

535 imposed by the forcing, this level of error is therefore the one expected for the modelled

536 wavelength and, in fact, it corresponds fairly well to the residual error for $N=20$ and 80 levels

537 (see Fig. 5).

538

In summary, the accuracy of the dispersion properties increases significantly with $N$

until ACM-related errors become small compared to the other error sources mainly the

540 vertical resolution and the order of precision of the numerical schemes used to calculate 
541 spatial and temporal derivatives. The determination of $N$ would lead to choosing $N$ around 14

542 in the case of the experiment considered. Another way to choose $N$ is to define an

543 "acceptable" precision level on the dispersion properties, depending on the period of the

544 simulated waves. For example, a relative error of $3 \%$ is obtained for $N>7$ for a period of $8 \mathrm{~s}$,

$545 \quad N>5$ for a period of 10 s, etc...

546

547

4.2 Improved accuracy (apart from increasing N)

548

549

Distribution of vertical levels

550

551

The previous paragraph shows that the accuracy decreases appreciably with the

552

wave period. At the shortest periods, accuracy is more sensitive to vertical resolution.

553

Increasing the number of vertical levels is an obvious but expensive way to reduce this

554 second source of error. Modifying the vertical distribution of levels without changing their number is an alternative that allows a significant improvement in accuracy at small periods. In the case of the 6 s period, Figure 5 shows that 10 irregularly distributed levels (see Table

1) provide a much higher accuracy than 10 equidistant levels, and makes it comparable to 558 the precision obtained with 20 equidistant levels. The vertical distribution proposed here is 559 such that the accuracy is also improved or maintained for the other periods considered 560 (results not shown).

561

\begin{tabular}{|l|l|}
\hline levels & Relative thickness $d z / h$ \\
\hline 10 & 0.03852 \\
\hline 9 & 0.04623 \\
\hline 8 & 0.05547 \\
\hline 7 & 0.06657 \\
\hline 6 & 0.07988 \\
\hline
\end{tabular}




\begin{tabular}{|l|l|}
\hline 5 & 0.09586 \\
\hline 4 & 0.11503 \\
\hline 3 & 0.13803 \\
\hline 2 & 0.16564 \\
\hline 1 & 0.19877 \\
\hline
\end{tabular}

Table 1: Thickness of the ten layers (1 corresponds to the bottom) as a fraction of the water depth.

564 It should be noted that, in a realistic domain, the generalized sigma coordinate makes it possible to adapt the vertical distribution to the bathymetry. For example, in areas where bathymetry is low relative to wave wavelength, the vertical profile of current and pressure is less sheared and a more regular distribution of the levels is preferable to the irregular distribution of Table 1. Given the positive influence of increasing vertical resolution on accuracy, the increase in vertical resolution that results from the decrease in bathymetry in the sigma formulation leads to a better representation of dispersion properties when approaching the coast.

572

573

Influence of horizontal resolution

574

575 Increasing the horizontal resolution (without changing the other parameters, 576 especially $N$ ) leads logically to decrease the level of error, especially at short periods. 577 Significantly increasing the horizontal resolution has a real but limited impact on the accuracy because of other sources of error such as the lack of vertical resolution. The period

$5796 \mathrm{~s}$ is more critical than the other periods tested because, as noted above, the number of grid 580 points per wavelength is particularly low. Doubling the resolution allows about 22 grid points 581 per wavelength (for $h=50 \mathrm{~m}$ ). Fig. 4 shows that a relative error $<3 \%$ is obtained with $\mathrm{N}>10$ for

582 a horizontal resolution of $2.5 \mathrm{~m}$, whereas this same level of error requires $\mathrm{N}>14$ for a 583 horizontal resolution of $5 \mathrm{~m}$. 
586 A modification of the pressure gradient scheme is now proposed to use moderate $N$

588

589

590

591

592

593

594

595

596

597

598 values while maintaining an acceptable level of accuracy on dispersion properties. We consider values of $N$ around 5. The proposed modification is suggested by the observation that the error on the wavelength is systematically negative (Figs 4 and 5). This is equivalent to the fact that the modelled phase speed is systematically lower than the theoretical phase speed. In other words, the non-hydrostatic effect excessively decreases the phase speed. This conjecture can be interpreted as an overestimation of the non-hydrostatic component of the pressure gradient in agreement with the conclusions drawn from the analytical solution of the linearized ACM equations (section 3, Figure 1). The proposed improvement consists in moderating the non hydrostatic pressure gradient by multiplying the latter by a constant $r$ slightly less than 1 (see the non-hydrostatic pressure gradient terms in equations (7) and $(8))$.
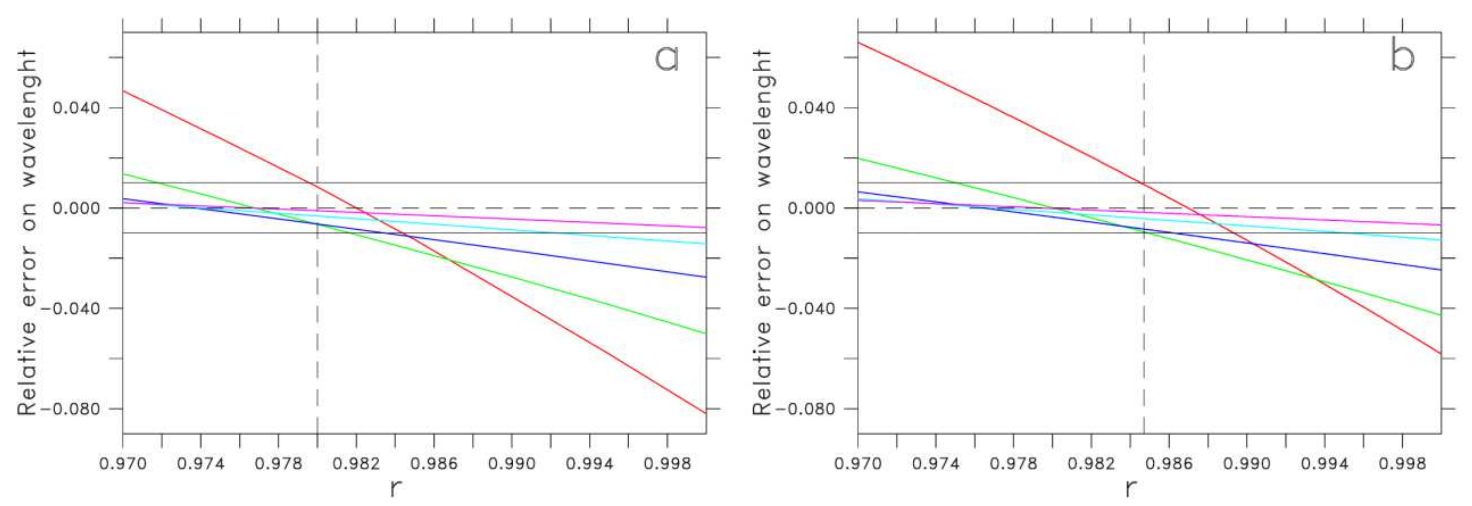

599

600

601

602

603

604

605
Figure 6: Relative error on the wavelength as a function of $r$. Model (a) and ACM linear theory (b). $h=50 \mathrm{~m} . \mathrm{N}=5$. Horizontal black lines indicate a relative error of + or $-1 \%$. Each curve corresponds to a period: $14 s$ (magenta), $12 s$ (cyan), 10s (blue), 8 s (green), 6s (red). The dashed vertical line corresponds to $r=0.98(a)$ and $r=0.9845(b)$ for which all relative errors are between $+/-1 \%$ 
606 Fig. 6a, obtained with $N=5(d t=0.032 \mathrm{~s})$, shows that the precision is improved when $r$

607 decreases to a certain level below which the sign of the error is reversed (becoming positive) 608 and the error increases again. As expected from the ACM linear theory, the value of $r$ that 609 exactly cancels the error is not the same depending on the period. It is around $r=0.982$ for 610 the $6 \mathrm{~s}$ period and smaller for the other periods. The general behaviour is nevertheless quite 611 similar for all the periods and one can identify a unique value of $r$ which makes it possible to 612 reduce the error below $1 \%$ for all the periods (Figure $6 a, r=0.98$ ), which corresponds to a 613 very significant reduction of the error, especially for small periods (error of $8 \%$ and $5 \%$ for 614 periods of $8 \mathrm{~s}$ and $6 \mathrm{~s}$ respectively, with $r=1$ ). The general behaviour of the model is in good 615 agreement with the analytical solution (Figure 6b). However, since the model has other 616 sources of errors than those of the theoretical model, Figures $6 a$ and $6 b$ are not strictly the 617 same and the best compromise for the value of $r$ would be slightly larger according to (24), 618 around 0.984 (Figure 6b). Although the theoretical model could certainly be used for a 619 default setting of $r$, Figure 6 a shows that errors can be further reduced with an empirical 620 setting. This numerical estimate of the best compromise on $r$ is therefore used in the 621 following.

622 As pointed out previously, a realistic numerical domain is characterized by variable 623 bathymetry. As $50 \mathrm{~m}$ should be an upper limit of a near shore zone model in our future applications, we now verify that the value $r=0.98$, which reduces the error below $1 \%$ for all periods when $h=50 m$, remains relevant for lower values of the water depth. These tests are done keeping the same time step $(d t=0.032 \mathrm{~s})$ and $N=5$. Figure 7 , obtained for $h=20 \mathrm{~m}$, shows

627 a precision greater than the simulation with $h=50 m$, under the combined effect of the 628 increase of the vertical resolution (the same number of vertical levels distributed over a 629 shallower water column) and also the decrease in the non-hydrostatic effect caused by the decrease in the ratio between the horizontal scale and the vertical scale. If Fig. 7 suggests that the best value of $r$ would be around 0.978 (the minimum relative error is indeed close to this value for most of the periods considered), the proposed value of 0.98 for $h=50 \mathrm{~m}$ remains 
633 relevant for shallower depths because it reduces the relative error associated with the 634 different periods between $-0.25 \%$ and $0 \%$.

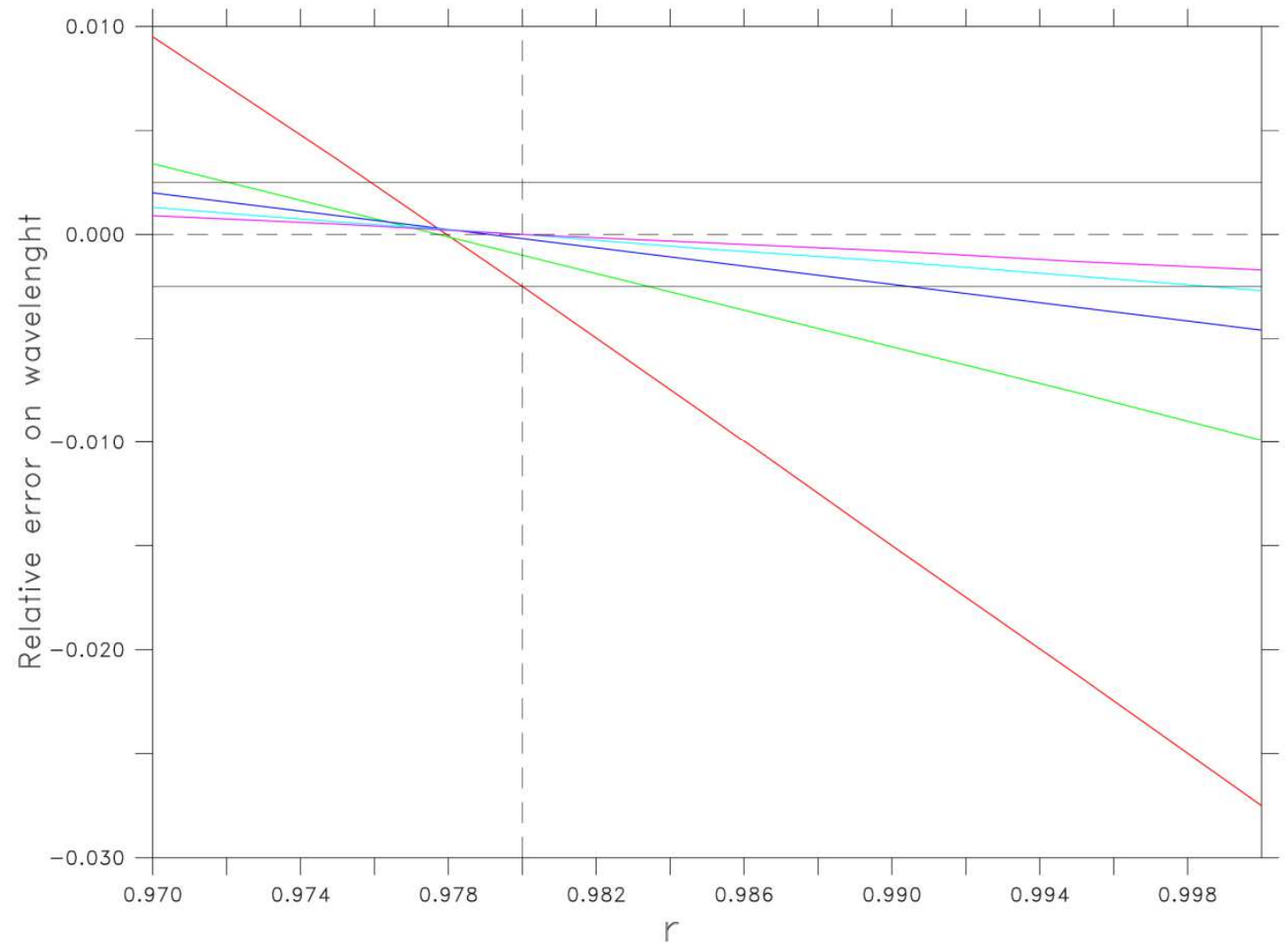

635

636 Figure 7: as Figure 6a but h=20m. The 2 horizontal black lines indicate a relative error of + or $6370.25 \%$. The dotted vertical line corresponds to $r=0.98$ for which all relative errors are between 638 $0.25 \%$ and $0 \%$.

639

640

Note finally that the choice of the $50 \mathrm{~m}$ value as the maximum value of the bathymetry 642 is somehow arbitrary. There are certainly other ways of defining the offshore limit of the near 643 shore zone, such as the depth at which the waves are modified by the seabed. In this case, 644 a maximum value based on the relative depth, $k h$, may be preferable. 
646 that this parameter can be used to correct errors beyond the single error caused by the ACM

647 method. The errors associated with the vertical discretization or the time-stepping scheme

648 can therefore be compensated by this method. We thus examine the possible undesirable 649 consequences of this compensating effect on other important properties of wave physics especially since, according to the theoretical model, the cancellation of the errors on the current and the relation of dispersion is not obtained strictly with the same value of $r$ (e.g.

652 (28) and (29)). Recall also that the shape of the vertical profile of the current is of particular 653 interest, since the intensity of the current near the bottom is crucial for the determination of 654 the bottom friction, the resuspension of sediments near the bottom, the transport of sand 655 (among others). Figure 8a shows that for the period 6s, $r=0.98$ leads to a vertical profile of 656 the current more accurate than with $r=1$ (the latter nevertheless correctly reproduces the vertical shear), the value $r=0.986$ being the one that would lead to a profile closest to the profile predicted by the linear non-hydrostatic incompressible theory. A similar conclusion is made for the period 10 s (Figure 8b) for $r=0.976$. The fact that a single value of $r$ can both reduce the error on the dispersion relation and the current is finally consistent with Figure 3 which shows that the values of $r$ cancelling these two types of error are actually very close.

662
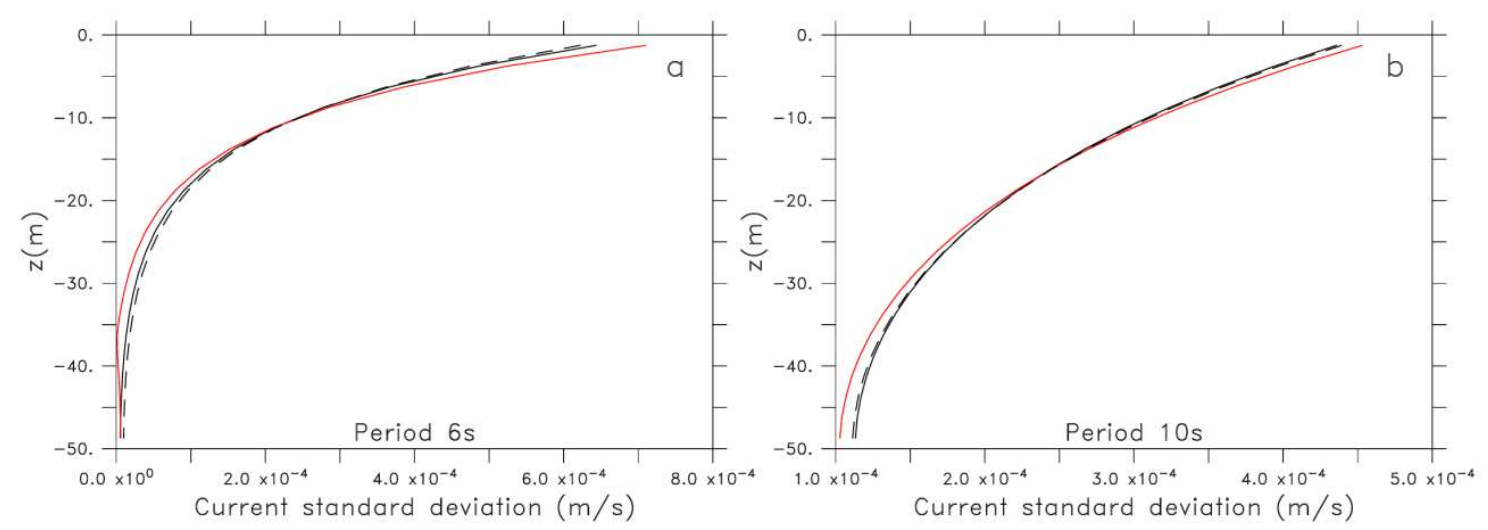

663

664 Figure 8: Standard deviation of the horizontal component of the current $(\mathrm{m} / \mathrm{s})$ as a function of the 665 depth $(\mathrm{m})$ calculated over the last 4 simulated periods at a distance of $625 \mathrm{~m}$ (125 points) from the 
incoming boundary. The period is $6 s$ (a) or $10 s(b) . h=50 \mathrm{~m}, 20$ vertical levels, $d x=5 \mathrm{~m}, N=5, r=1$

667 (red line), $r=0.98$ (black dotted line), theoretical profile from the incompressible linear theory 668 (continuous black line). For the sake of clarity the profiles corresponding to $r=0.986$ (period $6 s$ ) and 669 to to $r=0.976$ (periode 10s) are not shown because they merge with the theoretical profiles.

670

671

\subsection{Test case with bi-periodic domain}

673

We are now replacing open and closed boundary conditions at both ends of the domain in the propagation direction by periodic conditions. The interest of this bi-periodic domain is that it avoids reflections at the boundaries that could degrade the results. The simulations can therefore be long, which is of interest for testing the stability of calculations in the long term. The depth is $50 \mathrm{~m}$. The domain has 510 points in the direction of

679 propagation corresponding to a whole number of wavelengths, here 17 , for a wavelength of $680150 \mathrm{~m}$, a theoretical period close to $10 \mathrm{~s}$ and a phase speed close to $15 \mathrm{~m} / \mathrm{s}$. The fields are initialized from the linear theory. The amplitude of the surface elevation is small $(1 \mathrm{~mm})$ to remain within the framework of the linear theory. Nonlinear dissipation forces such as friction (using a quadratic parameterization) or breaking are therefore negligible. In this particular case, the Laplacian temporal filter derived from Marsaleix et al. (2012), used in the equations for $u, v, q$ to suppress the numerical mode, is the main source of dissipation. The following test quantifies its adverse effect on the long-term physical mode. Figure 9 shows the amplitude of the surface elevation at different times. Given the value of the phase speed, a distance of the order of $10 \mathrm{~km}$ (cross shore size of realistic domains that we plan to use with this model) is covered in about ten minutes. Figure 9 shows that the attenuation is negligible over such a short time duration. An attenuation of $10 \%$ would require at least 6 hours, during which the waves would travel a distance of about $300 \mathrm{~km}$. In our future realistic applications,

692 the open boundary condition will not be located so far from the coast. The cross-shore 693 domain dimension should be at least an order of magnitude lower, so this numerical 
694 attenuation should be less than 1\%, in other words small compared to the expected erosion 695 induced by bottom friction, turbulence or wave breaking.

696

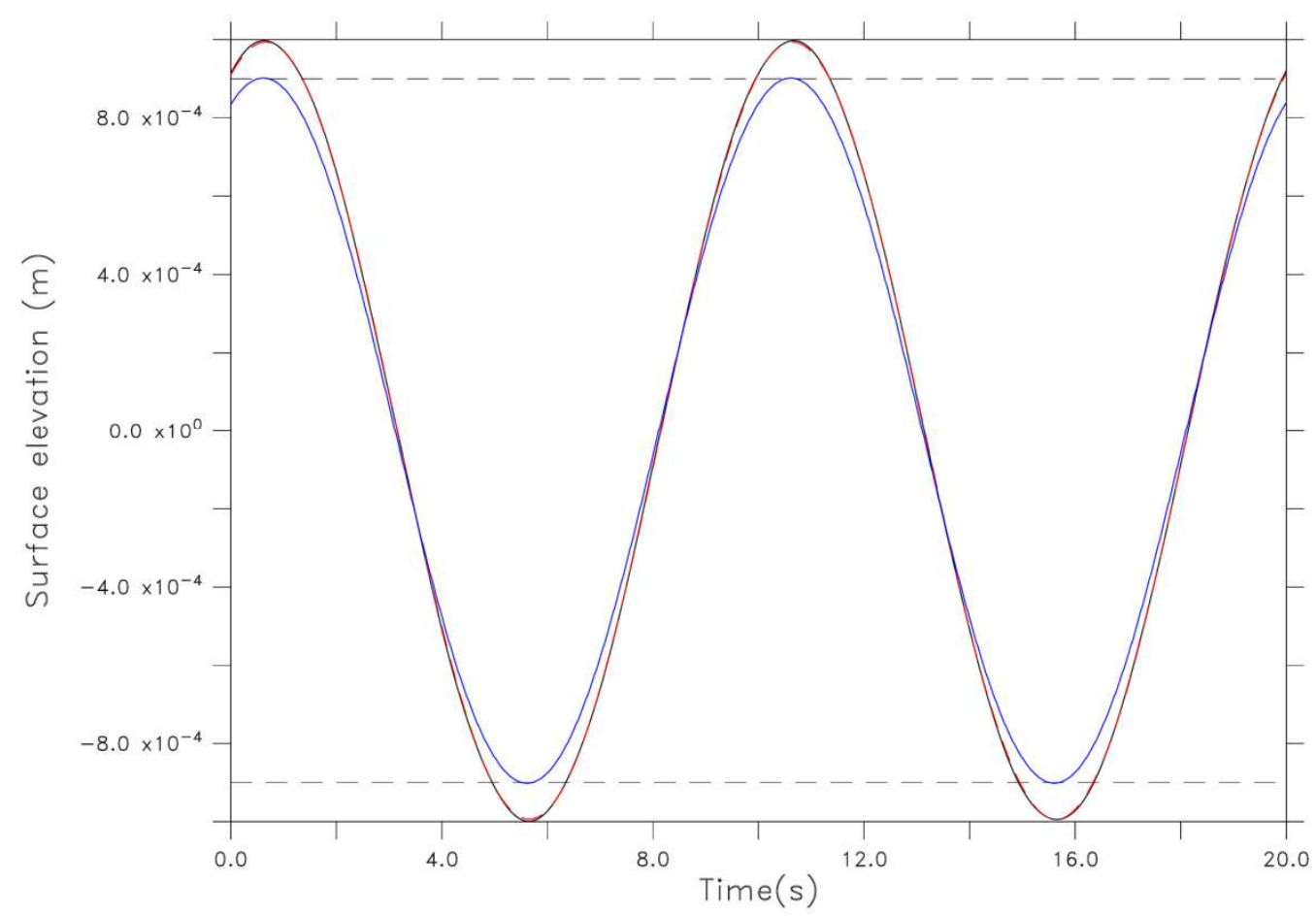

697

698 Figure 9: Surface elevation $(m)$ as a function of time $(s)$ at the beginning of the simulation (red 699 curve), after 10mn of simulation (black dotted curve), after 6 hours of simulation (blue curve). $700 h=50 m, d x=5 m, 20$ vertical levels, $N=5, r=0.98$. The 2 horizontal black dotted lines indicate an 701 attenuation of the initial amplitude of $10 \%$ 
707 hereafter HE01, Svendsen et al 2003) focuses on the propagation of waves over a uniform 708 linear slope beach, their breaking, and the alongshore current establishment. This case test 709 is of particular interest to us to assess the performances of the model on the important 710 question of the parametrization of wave breaking. Incoming waves are monochromatic, with 711 a period of $2.5 \mathrm{~s}$ (TEST $6 \mathrm{~N}$ in HE01). The amplitude of the excited waves at the open 712 boundary is adjusted to obtain a wave height corresponding to the conditions of TEST $6 \mathrm{~N}$, 713 namely $0.19 \mathrm{~m}$ at the most offshore measurement points (see the observation at 3 meters 714 from the incoming boundary on Fig. 10). The direction of propagation is $10^{\circ}$ from the 715 direction normal to the beach. A description of the basin and its bathymetry is given in 716 Svendsen et al. (2003) (see also Fig. 10). The particularity of the basin lies in its pumping 717 and recirculation system made of 20 pumps that allow having periodic conditions on the 718 lateral boundaries. In our simulation, incoming waves are specified from the linear theory at 719 the offshore boundary. Periodic boundary conditions are applied at the lateral boundaries of 720 the model. The dimension of the domain in the direction parallel to the beach is consistent with the condition of periodicity and the wave propagation direction of $10^{\circ}$ in the deep part of the basin. The horizontal resolution is $0.1 \mathrm{~m}$ and the grid has 10 vertical levels. The dimension in the cross-shore direction is $18 \mathrm{~m}$ and the deepest bathymetry, offshore, is $0.66 \mathrm{~m}$. The maximum wavelength, in the deep part of the basin, is of the order of $6 \mathrm{~m} . \mathrm{A}$ steady state is reached after about 150s. The results presented are obtained at the end of a simulation of 500 s. The roughness of the bottom corresponding to this experiment is a priori not mentioned by its authors. A roughness length of $3 \times 10^{-5} \mathrm{~m}$ was empirically fixed in our model in order to obtain a satisfactory agreement with the vertical profile of the measured alongshore current. The basin dimensions are small compared to a real case. If the ratio of the horizontal scale on vertical scale is used to deduce the characteristics that this experiment would have with dimensions 50 times larger, close to the dimensions of the test cases presented in the previous section, we obtain a horizontal resolution of $5 \mathrm{~m}$, a maximum depth of $30 \mathrm{~m}$, a 
maximum wavelength of the order of $300 \mathrm{~m}$ and a period of $125 \mathrm{~s}$. We are thus in conditions for which the problems of accuracy examined in the preceding sections are minor and make it possible to use the ACM method with a small value of $N$ and a coefficient of reduction of the gradient of $q$ close to one. In practice we use $N=4, r=1$ (as mentioned previously, we actually chose this test case for the wave breaking issue rather than the non-hydrostatic specificities discussed in the previous test case).

Wave breaking is parameterized through the horizontal diffusion of the momentum (13). The determination of the mixing coefficient, $v_{b}$, is an important aspect of this parameterization. Before going into the details of its calculation, we examine the order of magnitude necessary for the decay of the waves inside the breaking zone. To do this, we consider an energy balance between the gradient of the potential energy flux $\frac{\partial(g u \eta)}{\partial x}$ and

745 dissipation due to horizontal diffusion $-v_{b}\left(\frac{\partial u}{\partial x}\right)^{2}$ (for the sake of clarity we limit ourselves to unidirectional propagation along the $O x$ axis). We hypothesize that within the wave breaking zone the wave height is proportional to the water depth (Miche, 1944):

750

where $H$ is the wave height. We consider here that the parameter $\gamma$ is a constant of the order of 0.8 (it can nevertheless be noted that the expression of $\gamma$ an be more sophisticated, as in Guérin et al. 2018). For convenience (and considering that shallow depths, where breaking mostly occurs, allow this approximation) the hypothesis of hydrostatic equilibrium is made in the following reasoning (although the simulations presented are still non-hydrostatic). We thus consider that the current and the surface level are connected to each other according to $u=\frac{g}{c} \eta$ where $c=\sqrt{g h}$. It is finally assumed that the fields have a sinusoidal behaviour and

758 that the wave amplitude $\eta_{0}$ satisfies (32) (ie $\frac{2 \eta_{0}}{h}=\gamma$ ). Averaging over a period of waves, the 759 gradient of the potential energy flux is (APPENDIX D): 
$762 \quad \frac{\partial(g u \eta)}{\partial x}=g^{3 / 2} \gamma^{2} \frac{3}{16} h^{1 / 2} \frac{\partial h}{\partial x}$

763

764

and dissipation due to horizontal mixing is

765

766

$\varepsilon_{b}=-v_{b} \frac{1}{8}\left(\frac{k g \gamma h}{c}\right)^{2}$

767

768

The equilibrium of (33) and (34) leads to an estimation of the average of the mixing 769 coefficient over a period of waves:

770

$771<v_{b}>=\frac{3}{8} g^{3 / 2} h^{1 / 2} \frac{\partial h}{\partial x}\left(\frac{T}{\pi}\right)^{2}$

772

773 The latter increases with the slope of the bathymetry and vanishes when the thickness of the 774 water column is zero. The expression (35) is only an intermediate step towards a more 775 complete formulation taking into account a triggering criterion based on the analysis of the 776 instantaneous modelled fields. But before addressing this aspect, a preliminary experiment 777 is carried out to test the relevance of the order of magnitude given by (35). Practically (35) is 778 used as the value of the horizontal diffusion coefficient in (13) inside the wave breaking 779 zone. The latter is arbitrarily defined from a threshold on bathymetry $(h<0.28 m)$ deduced from observations of wave heights (HE01). In the pre-breaking zone $(h>0.28 m) v_{b}=0$. Figure 10 shows the simulated wave height and mean elevation, and the corresponding observations of HE01. As the laboratory experiment, the simulation lasts 500 s. The surface height is averaged over the last 50 seconds of the simulation. The wave height is calculated from the

784 difference between the maximum and minimum values of the surface level over the last 50 785 seconds of simulation. The agreement is satisfactory. The wave breaking trigger point is 
unsurprisingly well positioned since it is imposed by the criterion $h<0.28 m$. The wave height

787 is slightly overestimated in the breaking zone but the slope of decrease is parallel to that of 788 the theoretical decay $\frac{2 \eta_{0}}{h}=\gamma=0.8$ (dotted line in Fig. 10). It should be noted that several 789 simulations were performed by increasing or decreasing slightly the value of $\left\langle v_{b}\right\rangle$ and 790 that Figure 10 corresponds to a $5 \%$ increase in the value predicted by (35).
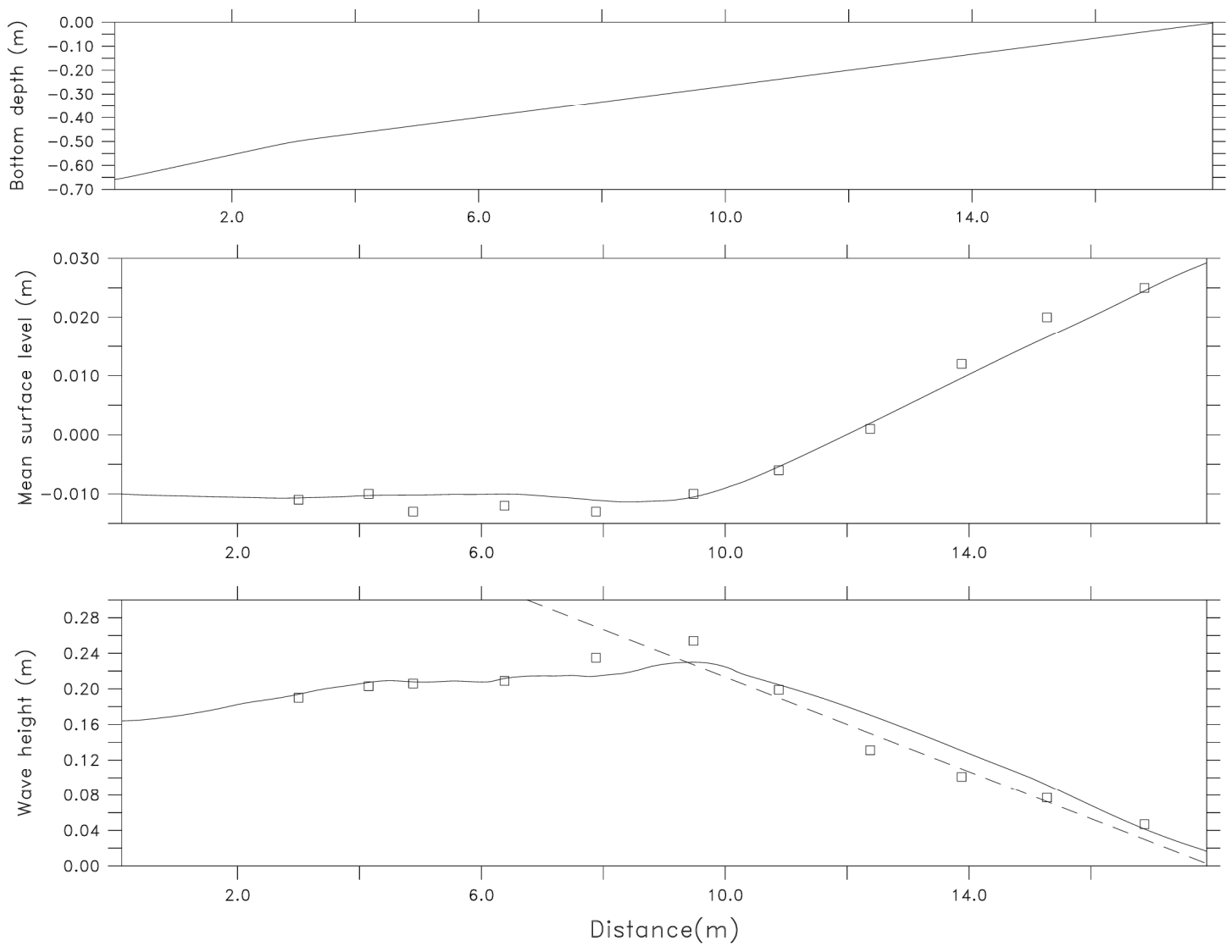

792

793 Figure 10. Top: Bottom depth (m). Middle: mean surface levels (m). Solid line: simulation. Squares:

794 longshore-averaged observations from HE01. Bottom: wave height $(\mathrm{m})$. Solid line: simulation. 795 Squares: longshore-averaged observations from HE01. Dashed line: 0.8h. Horizontal axis: crossshore distance from incoming boundary in meters. 
Breaking criterion

800

Practically (35) is not really appropriate because it requires to arbitrarily specify the

802

location of the onset of breaking. In realistic case, the breaking point of the breaking wave

803 varies over time, in particular according to the characteristics of the waves and more generally according to the surrounding hydrodynamic conditions. An extension of (35) taking into account the instantaneous fields is therefore proposed:

806

$807 v_{b}=<v_{b}>f_{b}$

808

809 Where $v_{b}$ is the instantaneous coefficient, $\left\langle v_{b}\right\rangle$ is the average coefficient (35) and $f_{b}$ is a 810 function that triggers the wave breaking according to the instantaneous fields. The average 811 of $f_{b}$ over a period of wave is expected to be close to 1 so that the viscosity is on average 812 close to $\left\langle v_{b}>\right.$. One consequence of this property is that $v_{b}$ can be momentarily very high if

$813 f_{b}$ is negligible over a large fraction of the wave period (so that the average of $v_{b}$ stays close

814 to $\left\langle v_{b}>\right.$ ). Many authors use the ratio of the wave height to the water depth (the $\gamma$ constant 815 (32)) as breaking criterion (Rusu and Soares, 2012). Others use criteria based on the 816 horizontal gradient of the modelled fields (Lynett, 2006). For example, Zijlema et al (2011) 817 propose to formulate eddy viscosity from Prandtl's length and a turbulence equilibrium 818 hypothesis which in practice links eddy viscosity to the horizontal gradient of velocities (see 819 their equation 12). Roeber and Cheung (2012) also use the velocity gradient (see their 820 equation 70) but in an approach that is more akin to a criterion on the Froude number, with $\sqrt{g h}$ as an approximation of the phase speed. These authors emphasize that this criterion has the particularity to be more influential in areas where the slope of the waves is important in contrast to the $H / h$ criterion which favours the wave crests. The criterion on the gradient

824 is therefore in principle influencing a larger proportion of the breaking zone. From the point of 
825 view of (36), an approach of this type should lead $f_{b}$ to act on a larger time interval and thus

826 limit the maximum values of $v_{b}$. On the other hand, a criterion on the wave crest should lead

$827 f_{b}$ to be small over a large fraction of the wave period, with the aforementioned consequence 828 of momentarily strong $v_{b}$ values. This consideration may be of numerical importance as 829 there is a stability criterion which limits the eddy viscosity value for a given time step (Zijlema 830 et al, 2011). In the particular case of this experiment, we find that a wave breaking criterion 831 based on $H / h$ can cause the eddy viscosity to momentarily exceed the maximum value 832 allowed by the CFL criterion (ie $v_{b}>v_{b}^{\max }=\frac{1}{4} \frac{d x^{2}}{d t}$ ). One possibility of overcoming this 833 problem is either to reduce the time step of the model, or to use a time splitting technique with a smaller time step for the calculation of the wave breaking without impacting the time step of the other equations of the model. From a numerical point of view, the approach proposed by Roeber and Cheung (2012) is interesting because their parameterization of the wave breaking should act on a longer duration, limiting the maximum values of the breaking viscosity. We choose this approach, mainly for the aforementioned numerical issues, but we propose to add a modification to that method in order to extend the influence of the breaking criterion to an even greater proportion of the breaking zone. This is arguably questionable from the point of view of the realism of the process (Kennedy et al, 2000) but it is an

842 interesting option considering the numerical aspects mentioned above. More specifically, our

843 objective is to avoid reducing the time step because of a too restrictive stability condition 844 related to the wave breaking scheme.

845 The triggering criterion for wave breaking is inspired from Roeber and Cheung (2012) 846 but is modified to be active both on the wave crest and between crests. This criterion 847 considers both velocities and their horizontal gradient. The velocity gradient is scaled by the 848 wavenumber so that the two quantities have the same order of magnitude. A wave breaking 849 criterion is then given by the ratio of the sum of these fields to the hydrostatic phase speed 850 (for simplicity the following formula is given in the case of unidirectional propagation): 
$852 f_{b}=c_{1}\left[\frac{u_{s}^{2}+\left(\frac{1 \partial u_{s}}{k \partial x}\right)^{2}}{g h}\right]^{n / 2}$

853

854 Where $u_{s}$ is the surface current and $k$ is the wavenumber fixed a priori according to the 855 peak period prescribed at the open boundary. The constant $c_{1}$ is determined empirically: 856 ideally, its role is to allow the average value of $f_{b}$ to be of the order of unity over a period of 857 waves (so that the eddy viscosity is on average close to (35)). It may be noted that in the 858 idealized framework of the linear theory (where the current would be of the form $u_{0} \cos (\omega t+$ $859 k x)$ ) the term in the numerator of (37) would simply be $u_{0}$, independent of time, and, in a 860 breaking situation characterized by a current of an order of magnitude comparable to that of 861 the phase speed, the constant $c_{1}$ would simply be of the order of (35). The exponent, $n$, is 862 used to adjust the selectivity of the criterion. It is in particular chosen so that $f_{b}$ tends more 863 rapidly towards 0 upstream of the breaking zone. The simulations presented in the following 864 use $n=2$.

865

866 

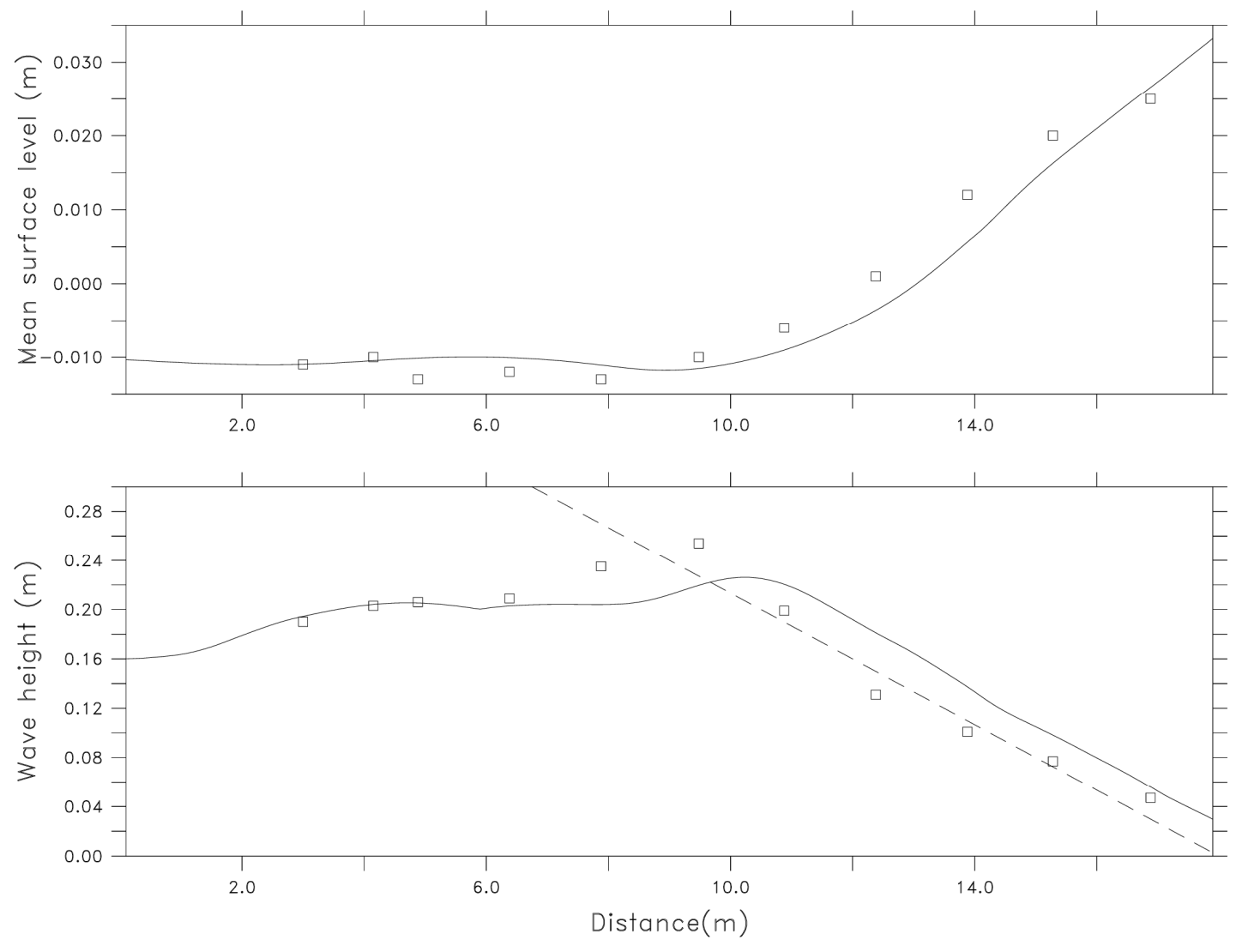

867

868 Figure 11. As Figure 10 but eddy viscosity calculated with (36) and (37). Top: mean surface level 869 (m). Bottom: wave height (m). Dashed line: 0.8h.

870

871

872 Figure 11 corresponds to an eddy viscosity calculated with (36) and (37) instead of (35) with

873 the arbitrary hypothesis on the depth of the breaking onset (the other parameters being 874 identical to those of the previous simulation). It is comparable to Fig. 10. The main difference 875 is in the behaviour within the breaking zone where the curves are a little less linear in the 876 case of Fig. 11, as a consequence of the non-linear character of (36) and (37). Although the 877 simulation is slightly less close to the observation in the case of Fig. 11, we prefer to use 878 (36) and (37), whose formulation is more general rather than (35) which suffers from its 879 arbitrary definition of the trigger point of breaking. 
881 Taking into account the breaking in the turbulent closure scheme

882

883 The turbulence scheme used here is the k-epsilon scheme (Rodi, 1987). A wave884 related turbulence generation term may be taken into account when the circulation model is 885 coupled to a phase-averaged wave model (Michaud et al, 2012). In the present case, this 886 term is not taken into account because the explicit modelling of the waves leads naturally to 887 a significant production of turbulent kinetic energy (TKE) through the vertical shear of the 888 current. The relevance of adding an additional production term to represent the assumed 889 increase in turbulence in the breaking zone is an open question (Feddersen and Trowbridge, 890 2005) and is therefore examined here. The HE01 experience provides the opportunity to examine this point by providing measurements of the residual onshore current at different depths and at different distances from the edge of the basin. Here, an additional production

893 term, $P_{w b}$, representing a fraction of the kinetic energy lost by the current via the horizontal 894 mixing associated with the breaking (ie (36), (37)), is introduced into the TKE equation of 895 the k-eps scheme:

896

$897 \quad P_{w b}=c_{2} v_{b}\left[\left(\frac{\partial u}{\partial x}\right)^{2}+\left(\frac{\partial u}{\partial y}\right)^{2}+\left(\frac{\partial v}{\partial x}\right)^{2}+\left(\frac{\partial v}{\partial y}\right)^{2}\right]$

898

899 Where $c_{2}$ is a constant between 0 and 1. Equation (38) implies that a fraction of the breaking 900 energy feeds the vertical mixing. The simulated alongshore residual current is obtained by averaging the alongshore current during the last 50 seconds of the simulation. Figure 12 shows the comparison with the observed current from HE01. Note that the vertical axis has been normalized so that the figure is easily comparable to that of Svendsen et al. 2003 (see their figure 11). Two simulations are made, one with $c_{2}=0$ the other with $c_{2}=0.01$. The two simulations generally give rather satisfactory results with the exception of the current at $x=7.9 \mathrm{~m}$. Looking more closely at Fig. 12 , we notice that the simulation taking into account 
$907 P_{w b}$ (red lines) gives a better agreement with the observations. The simulation without $P_{w b}$

$908\left(c_{2}=0\right.$, green lines) shows indeed a current with a greater vertical gradient than that of the

909 observation, in particular at the points located in the shallowest depths. Increasing the

910 vertical mixing coefficient associated with $P_{w b}$ reduces the current shear in the breaking

911 zone. We therefore retain at this stage that this approach can be used to improve turbulence

912 in the wave breaking zone. However, in the state of our knowledge, the setting of $c_{2}$ is

913 arbitrary and would need to be estimated on other experiments, including in situ data such

914 as current profiles or more direct observations of turbulence (Costa et al. 2017).
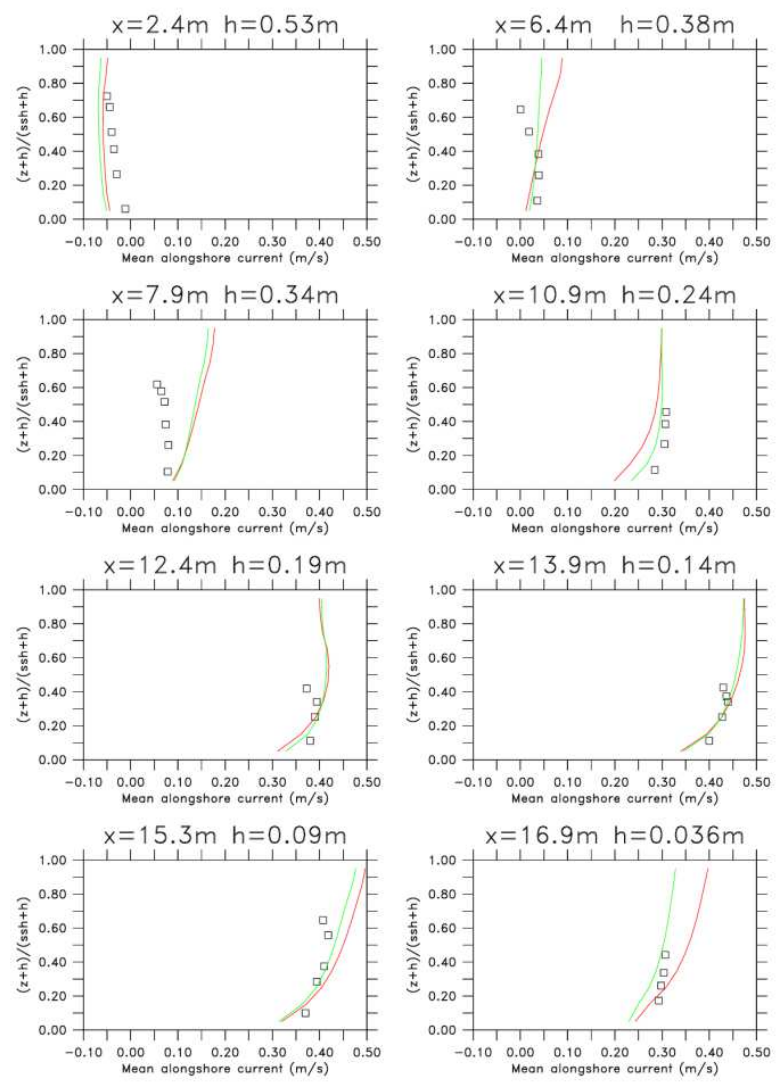

915

916 Figure 12. Vertical profile of the measured mean alongshore current (black squares), simulated

917 without the term $P_{w b}\left(c_{2}=0\right.$, green lines), simulated with the term $P_{w b}, c_{2}=0.01$ (red lines).

918 Vertical axis: normalized depth $\frac{z+h}{\eta+h}$. Horizontal axis: $\mathrm{m} / \mathrm{s}$.

919 

interactions between current and waves (Weir et al, 2011). The model is now applied to a laboratory experiment describing the propagation of monochromatic waves over a beach with a sandy bar and channels inducing rip currents (Haller et al, 2002, Haas and Svendsen, 2002). This experiment has already been simulated with hydrostatic circulation models coupled with a phase-averaged wave model (Haas and Warner, 2009, Haas et al, 2003, Michaud et al., 2012, Bruneau 2009). Like these previous authors, we are interested here in TEST B of Haller et al. (2002). The basin and its bathymetry are described in detail in the aforementioned articles. The dimensions of the basin are approximately $17 \mathrm{~m}$ (cross-shore direction) by $18 \mathrm{~m}$ (alongshore direction). The cross-shore slope is $1 / 30$. A bar, located a few meters from the coast, is cut by 2 channels. Ideally, the two channels should have an identical shape and the bathymetry of the entire basin should respect an alongshore symmetry: the central bar is twice as long as the two half-bars touching the side walls and outside the bar the alongshore gradient is supposed to be nil. However, as pointed out by Haas et al. (2003), an important feature of this bathymetry is that it actually has irregularities compared to its idealized design, resulting in dissymmetry of the flow and increasing instability of the currents. The two rip currents that form at the exit of the channels are for example clearly different in their form and their intensity, and their respective temporal variations seem rather decoupled. A bathymetry interpolated from a survey of the wave

941 basin is used here to better respect the real conditions of the experiment. The horizontal 942 resolution is $0.06 \mathrm{~m}$ and the vertical grid has 10 sigma levels. The time step is $1.1 \times 10^{-2}$ 943 seconds. Monochromatic waves of period 1s propagate in the direction perpendicular to the 944 coast (Fig. 13). The amplitude of the excited waves at the open boundary is adjusted to obtain a wave height corresponding to the conditions of TEST B, namely about $4.1 \mathrm{~cm}$ at the most offshore measurement points (see Fig. 14 around $x=8 \mathrm{~m}$ ). Their wavelength at the 947 entrance of the domain (where the depth is about $0.38 \mathrm{~m}$ ) is about $1.4 \mathrm{~m}$ (Fig. 13) in good 948 agreement with the linear non hydrostatic wave theory. It may be noted that the hydrostatic 

overestimation of about $35 \%$.

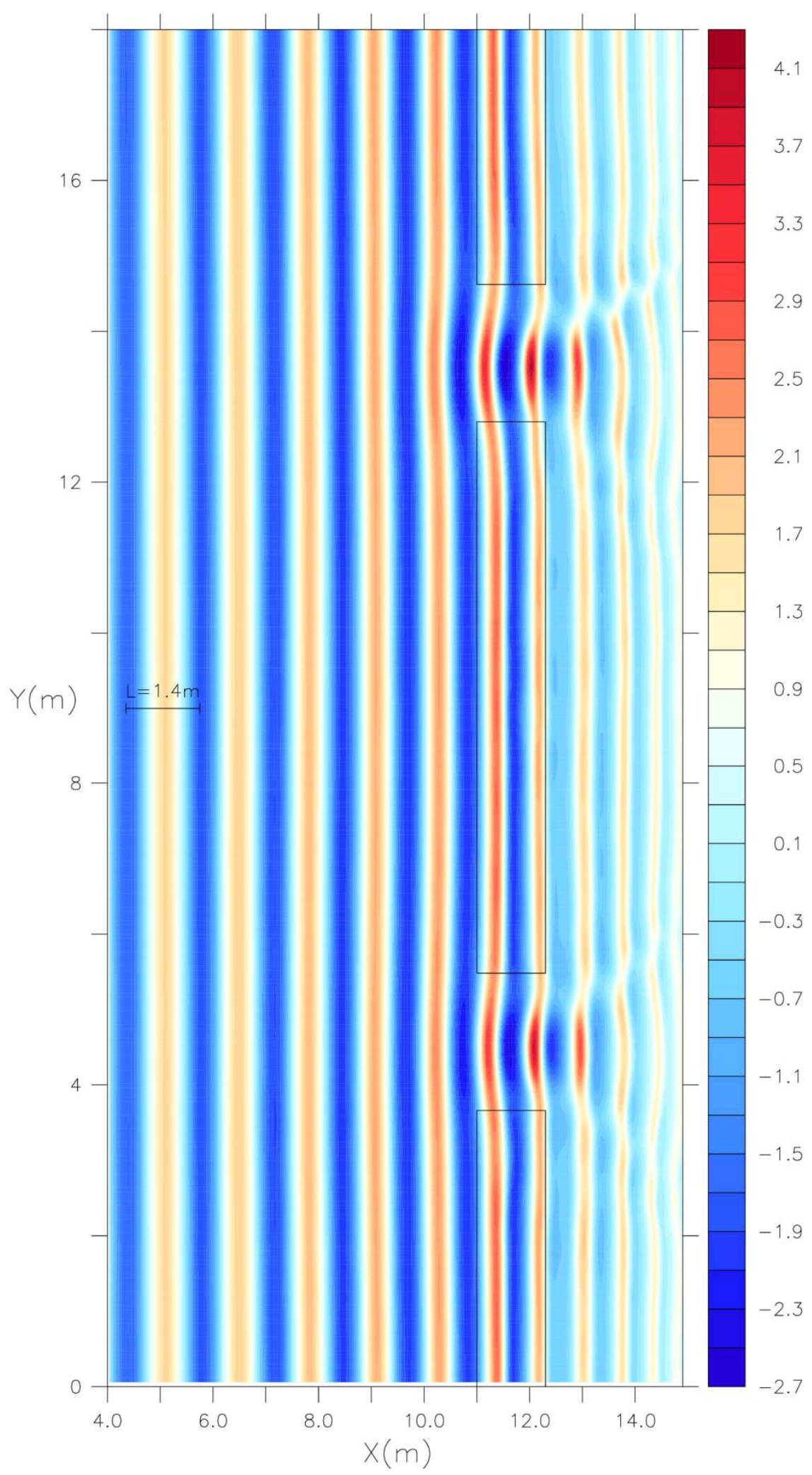


Figure 13. Surface elevation (cm) after 1 minute of simulation. black rectangles: position of bars.

953 Short black line with label: scale of the wavelength at the entrance of the basin. Horizontal axis:

954 cross-shore distance ( $m$ ) from the wave generator. Vertical axis: alongshore distance $(m)$

955

956

957 Consistently with Haas and Svendsen (2002) and Haas et al. (2003), the simulation lasts 30

958 minutes to highlight the observed temporal variations of rip currents. The wave height is

959 obtained from the difference between the minimum and maximum of the surface level

960 obtained over time intervals of 1 second. An average wave height is then calculated by

961 averaging these values obtained every second over the simulation except the first minute to

962 avoid the spin-up phase. Table 2 summarizes the characteristics of the numerical grid and

963 the calculation time. The latter can be roughly extrapolated to more realistic applications considering that the scale ratio between this simulation and that of a realistic case (with for example $d x=3 \mathrm{~m})$ is about 50 , in other words an area of about $1 \mathrm{~km} \times 1 \mathrm{~km}$ and a simulation duration of about 1 day.

967

\begin{tabular}{|l|l|}
\hline Number of grid points & $300 \times 284 \times 10$ \\
\hline Horizontal resolution & $0.06 \mathrm{~m}$ \\
\hline Horizontal size of the domain & $18 \mathrm{~m} \times 17 \mathrm{~m}$ \\
\hline Parallel calculation & 72 cores \\
\hline Duration of the simulation & 30 minutes \\
\hline Number of iterations & 1600000 \\
\hline Computing time & $10 \mathrm{~h}$ \\
\hline
\end{tabular}

968 Table 2: grid specificities and computing time on the OCCIGEN supercomputer administered by

969 CINES (https://www.cines.fr) 
972 the observations of the TEST B case (Figures 6 and 16 of Haller et al, 2002). The wave 973 height first increases slowly towards the coast, then increases sharply when the waves 974 arrive above the bar (Figure 14). The breaking conditions are quickly reached and once the 975 bar crossed, the wave height is much lower and relatively constant (Figures 13 and 14).

976
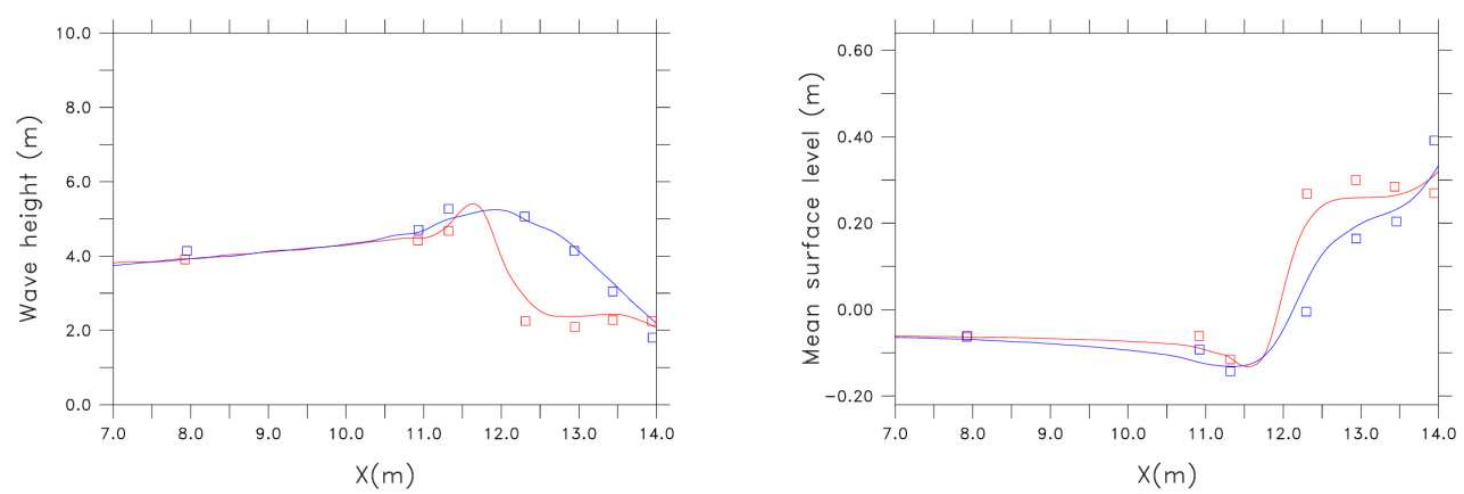

977

978

Figure 14. Left: Mean wave height (cm) as a function of cross-shore distance. Right: Average surface 979 elevation ( $\mathrm{cm}$ ) depending on the cross-shore distance. In red: in the center of the basin $(y=9 \mathrm{~m}$, with 980 bar). In blue: in the channel axis $(y=13.6 \mathrm{~m})$.

981

982 A second breaking zone is highlighted near the coast. This behaviour of the model is in good 983 agreement with the description of the process given in Haller et al. (2002). In the channel 984 axis, the wave height increases before the channel, as shown in Figures 13 and 14, because 985 of the blocking by the opposite rip current. Figure 15 shows the horizontal distribution of the 986 depth-averaged current, that is very similar to the circulation calculated with the phaseaveraged modelling presented by Haas et al 2003 (their figure 5) and Haas and Warner 988 2009 (their figure 7).

989 


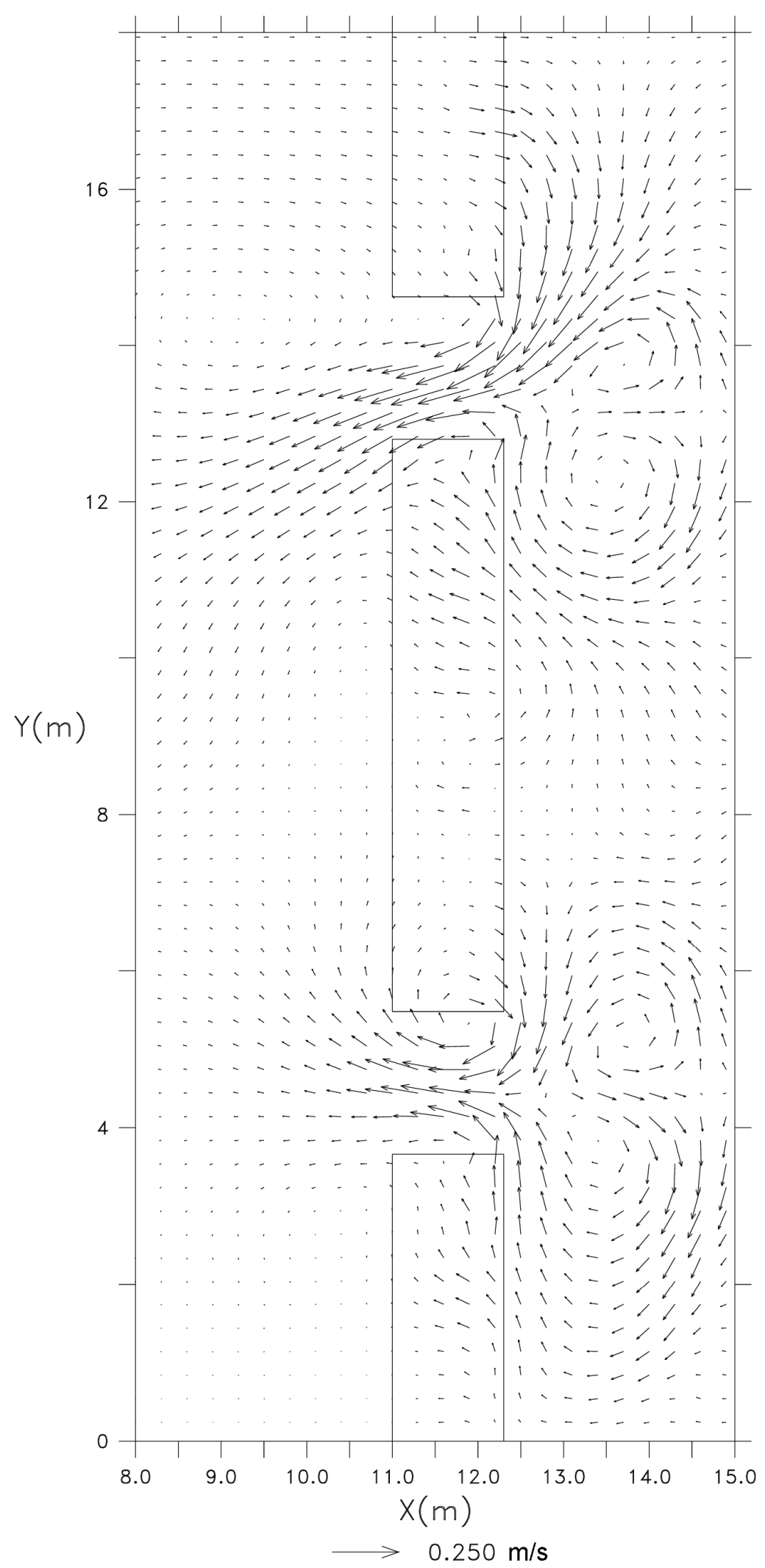


991 Figure 15. Depth and time averaged current $(\mathrm{m} / \mathrm{s})$ computed by SYMPHONIE. Horizontal axis:

992 distance $(m)$ cross-shore from the wave generator. Vertical axis: distance $(m)$ alongshore.
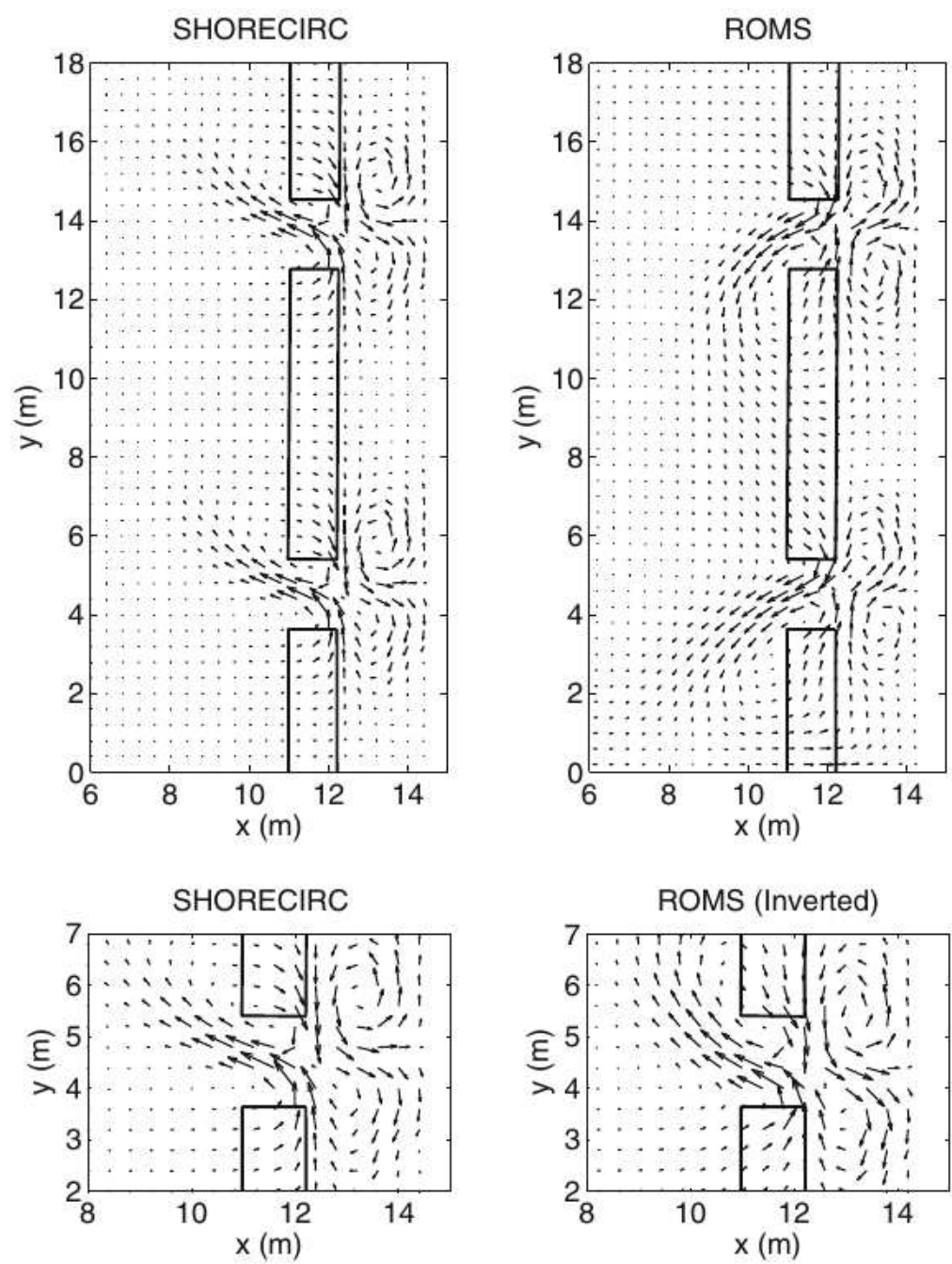

994 Figure 15 continued: Figure 7 in Haas and Warner (2009). Depth-integrated mean currents. 
(a)

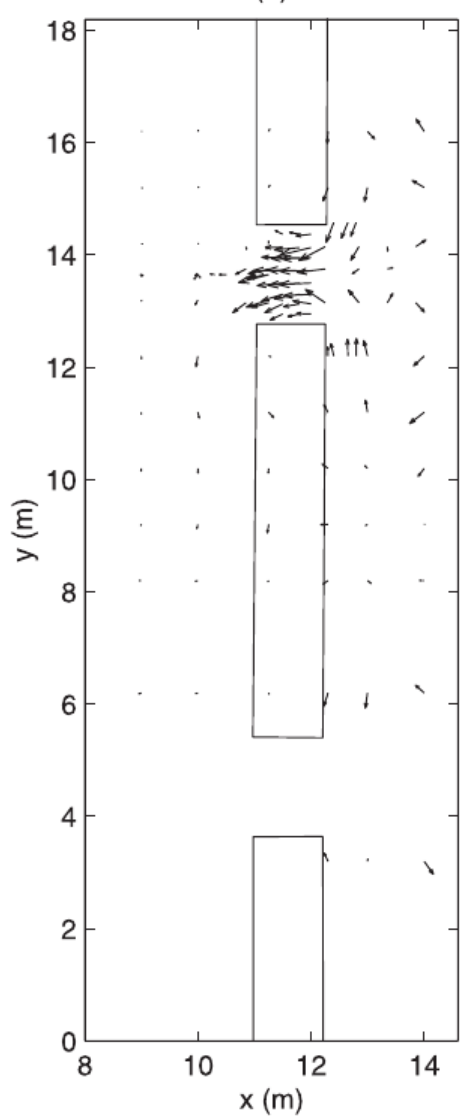

(b)

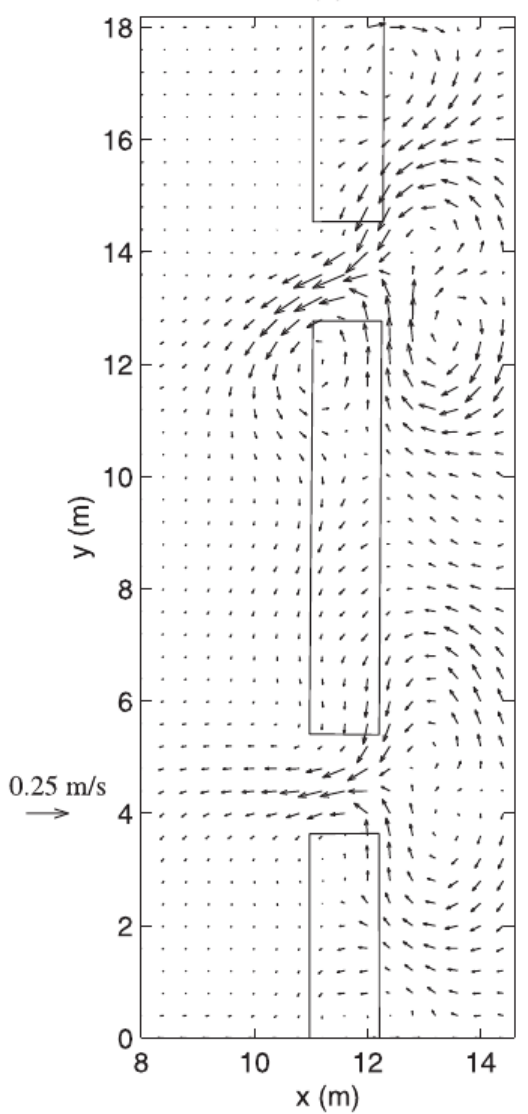

(c)

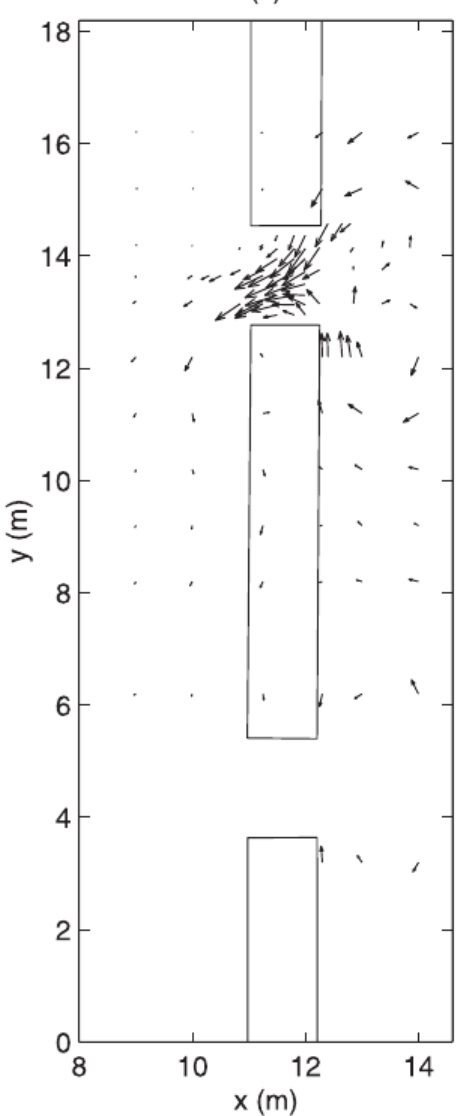

995

996

997

998

999

1000

1001

1002

1003

1004

1005

1006

1007

1008

1009

Figure 15 continued: Figure 5 in Haas et al (2003). Time-averaged depth-integrated current from (a) experimental data, $(b)$ the simulation, and (c) the simulation at the same points as the experimental data

The rip current slows down the waves, causing the waves to refract toward the centre of the channel (Figure 13), in agreement with the visual observation of the laboratory experiment reported by Haas et al. (2003), hereafter H03, (see their section 6). However, the rip current does not lead to a wave breaking more important than the one above the bar. The wave height actually decreases more slowly in the channel than above the bar, so that in the last meters before the coast the waves are higher in the channel axis than behind the bar (Figure 14). The mean surface level increases at the coast, inducing a change in the average position of the water line allowed by the wetting-drying scheme of the model. The mean surface level at the coast is markedly different in the channel axis and in front of the bar, in 
1010 agreement with the observation (Figure 14). As Haas and Svendsen (2002) point out, this 1011 difference is associated with a longshore pressure gradient that drives the flow to the 1012 channels. Laboratory experiments show that rip currents are unstable and are characterized 1013 by large temporal variations. The authors of these experiments emphasize the impossibility 1014 of reproducing twice the same series of measurements, even if the experimental conditions 1015 are a priori unchanged. An exact agreement with the model is therefore not sought. 1016 Nevertheless, we expect from the model that it gives the correct orders of magnitude of the 1017 rip currents and their variability. Figure 16 shows the temporal evolution of the current 1018 averaged over the vertical and over a wave period in the two channels. Given the above 1019 limitations, the agreement with the observations presented in $\mathrm{HO}$ (see Figure 11.a in H03) 1020 is good. The rip current at $y=4.6 \mathrm{~m}$ is a little overestimated but, as in H03, it is mostly lower 1021 than the rip current at $y=13.6 \mathrm{~m}$. The latter has the good order of magnitude, with maximum around $20 \mathrm{~cm} / \mathrm{s}$. The amplitude and time scale of its variability are also close to the 1023 behaviour described in $\mathrm{H} 03$.

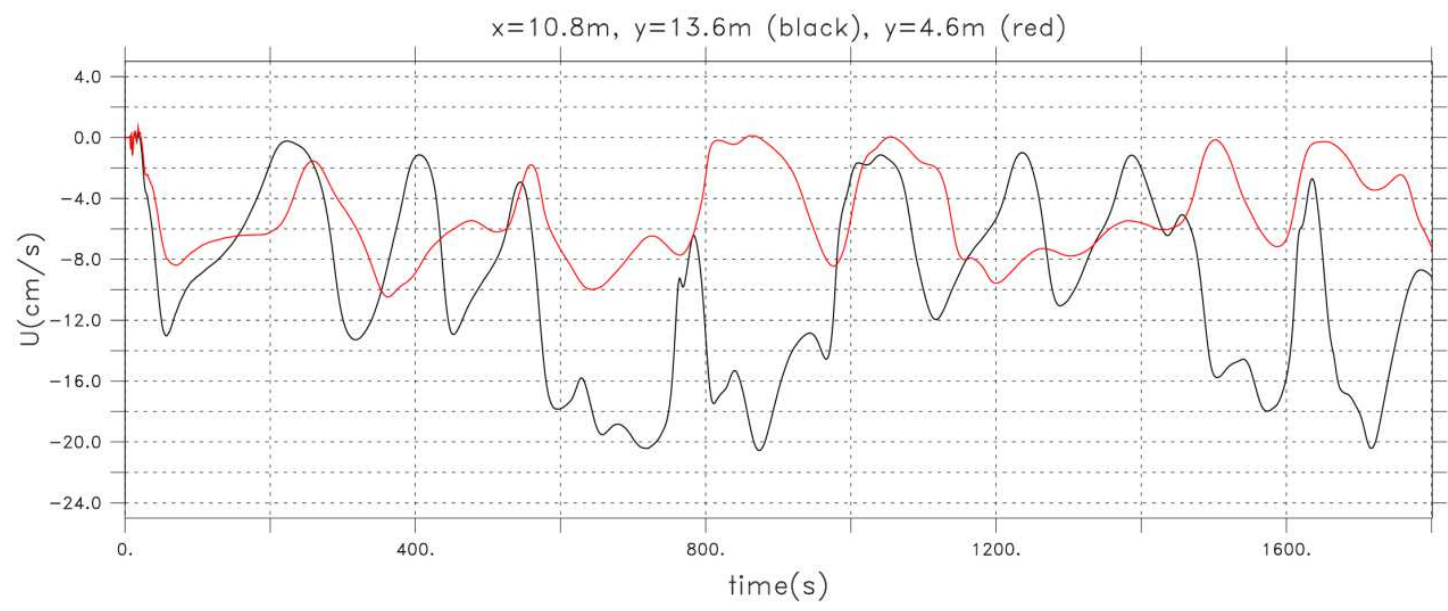

1024

1025 Figure 16. Cross-shore depth-averaged phase-averaged current $(\mathrm{cm} / \mathrm{s})$ in the two channels at $1026 x=10.8 \mathrm{~m}$ as a function of time (s). Periods shorter than $1 \mathrm{~s}$ are filtered. Negative values in the 1027 direction of the wave generator. $y=13.6 m$ (black line), $y=4.6 m$ (red line). 
1029 The occurrence of the rip current is of the order of 200 seconds without being really regular.

1030 The variability of the rip current is of the order of a few centimetres per second, with a 1031 maximum standard deviation around $6 \mathrm{~cm} / \mathrm{s}$ in the centre of the channel at $\mathrm{y}=13.5 \mathrm{~m}$ and $1032 \quad \mathrm{x}=11.5 \mathrm{~m}$ (Fig.17). 


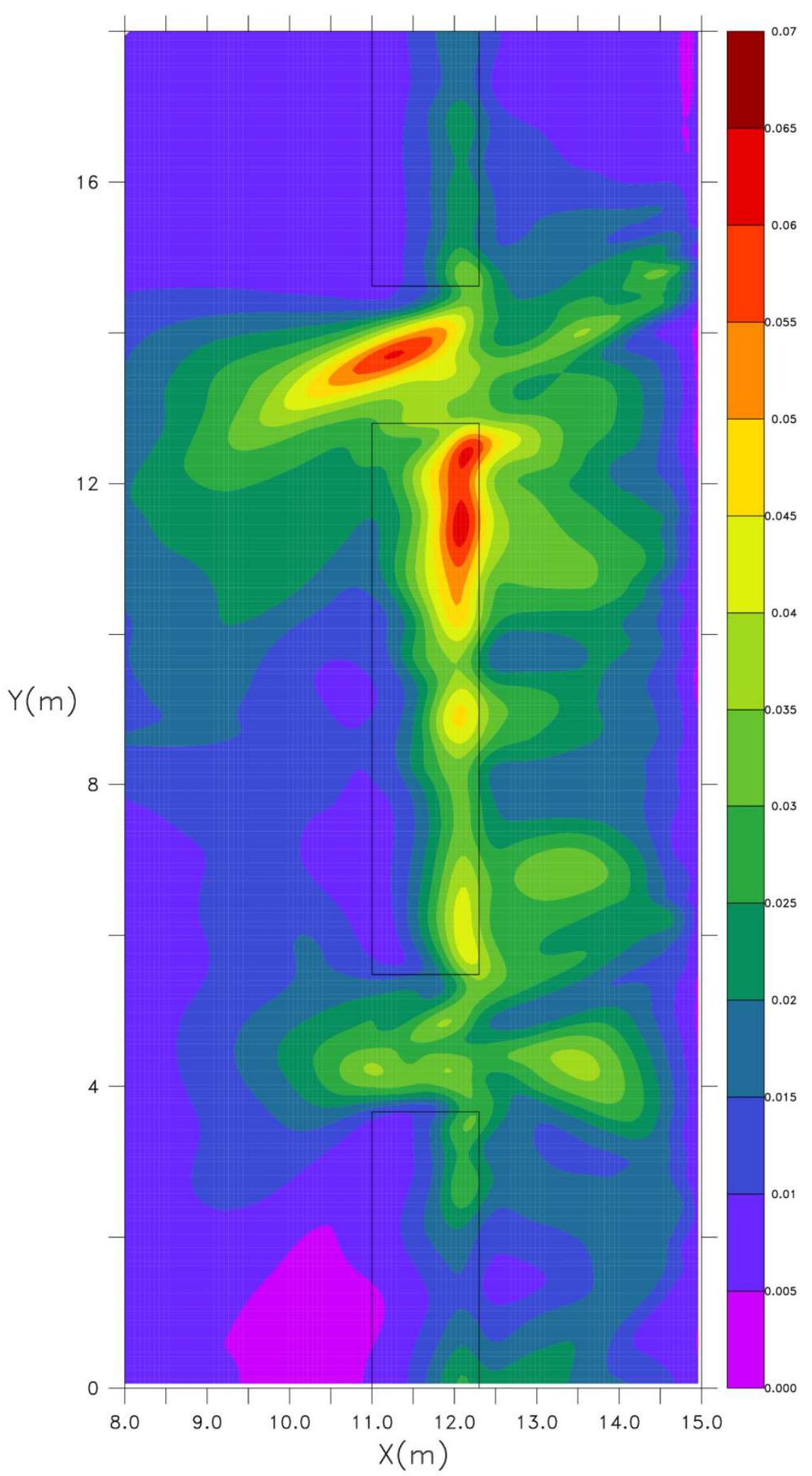

1033

1034 Figure 17. Standard deviation of the cross-shore depth-averaged current $(\mathrm{m} / \mathrm{s})$ 
1037 The vertical structure of the time-averaged cross-shore current in the rip channel (Fig.18) 1038 has similarities with the phase-averaged modelling of Haas and Warner (2009, their Figure 1039 11) with a maximum intensity current around $0.2 \mathrm{~m} / \mathrm{s}$ between $\mathrm{x}=11 \mathrm{~m}$ and $\mathrm{x}=12 \mathrm{~m}$. At the 1040 point of maximum intensity $(x=11.5 \mathrm{~m})$, the current is fairly homogeneous on the vertical 1041 except near the bottom where the current decreases strongly, in good agreement with the 1042 observation (Haas and Svendsen 2002, their figures 17, 18, 19). At the exit of the channel 1043 (offshore direction), the current decreases significantly. The vertical gradient also becomes 1044 larger and close to the bottom at $x=9 m$ the current is even reversed (positive values in 1045 Fig.18), in good agreement with the observations (Haas and Svendsen 2002, their figures 13 1046 and 14).

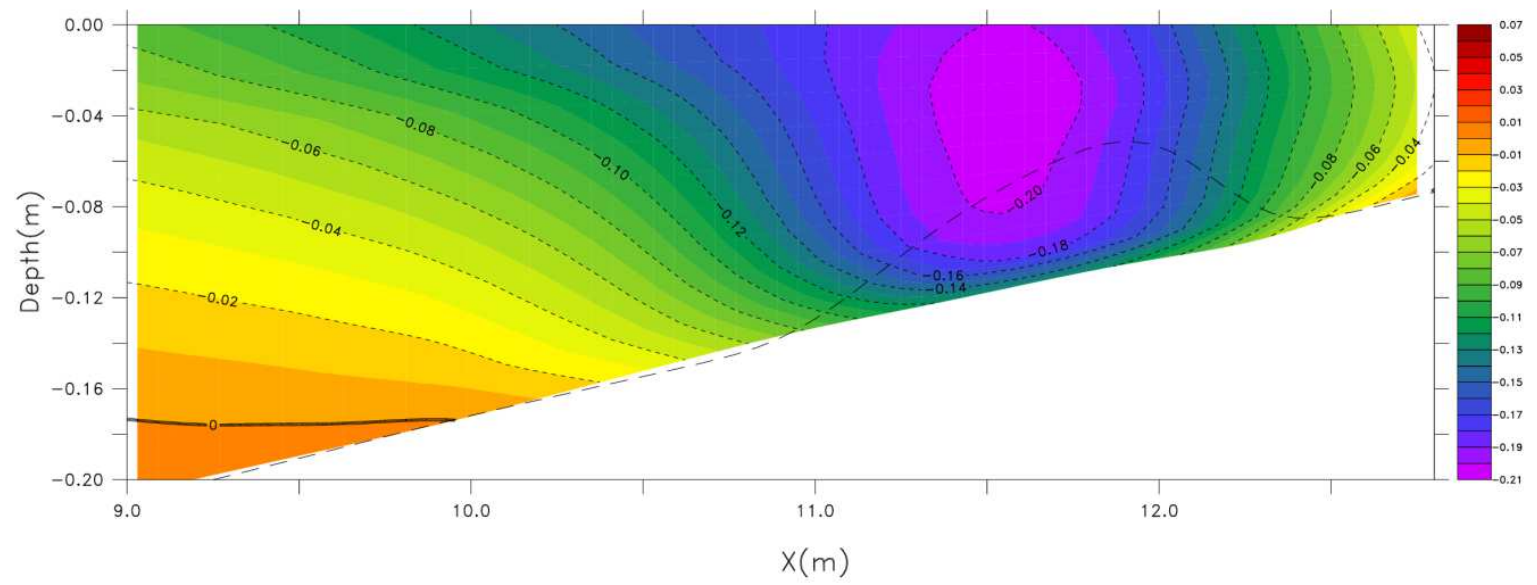

1047

1048 Figure 18. time-averaged cross-shore current $(\mathrm{m} / \mathrm{s})$ at $y=13.6 \mathrm{~m}$. Negative values are in the offshore 1049 direction (decreasing $x$ ).

1050

1051

\section{Conclusions}


A phase-resolved wave model is derived from an ocean circulation model with the

1055 aim of performing simulations of current-wave interaction in nearshore areas. The model is

1056 evaluated on the fundamental aspects of wave dynamics, namely mainly the dispersion

1057 properties, the transformation of wave characteristics by bathymetry (shoaling, wave1058 breaking), the influence of waves on low frequency fields such as the mean surface levels 1059 and rip currents.

1060 With the exception of the post-breaking zone that can be processed in some models 1061 by a hydrostatic approach, the phase-resolved wave modelling requires a non-hydrostatic 1062 model. As the representation of the non-hydrostatic problem in all its complexity seems to be 1063 inevitably very expensive to date (Klingbeil et al, 2018), a scheme allowing a reasonable compromise between, on the one hand the extra cost of the computation, and on the other hand an acceptable loss of accuracy of non-hydrostatic properties of waves, has been introduced into the circulation model. It is shown that relative errors of less than a few \% (on

1067 the dispersion relation and the vertical current profile) can be obtained with non-hydrostatic 1068 simulations costing 5 to 6 times more than a hydrostatic model calculated under the same conditions. The non-hydrostatic additional cost remains obviously high but low enough to consider simulations of realist events such as storms on very large areas and it is anyway worth noting that wave-current effect modelling based on a hydrostatic circulation model normally couple the latter with a phase-averaged wave model whose cost is possibly not negligible.

The non-hydrostatic method implemented in the circulation model is derived from the 1075 method proposed by Lee et al. (2006). In practice, the classical Poisson problem is 1076 transformed into a pressure propagation equation. The resolution is iterative, local, and does 1077 not need to solve a system of linear equations. An essential aspect of this method is to properly control the various parameters related to the iterative aspect of the problem. The 1079 equation of pressure propagation depends on a constant (the latter being comparable to a 1080 propagation velocity) which determines the speed of the adjustment of the non-hydrostatic 1081 pressure to a change of the hydrostatic terms in the momentum equations. The precision of 
the non-hydrostatic behaviour depends on the fact that this adjustment is faster than the

1083 variation of the other fields. A meticulous adjustment of the time step of the model is in 1084 practice necessary, taking into account various parameters, including the wave propagation speed and a possible tuning consisting in reducing the non-hydrostatic pressure gradient. So far the reduction factor is a constant finding of a compromise in a set of possible values taking into account a range of periods and depths. Future development will adapt this tuning to the wave peak period and bathymetry using the analytical reduction factor solution that this study established from the linearized ACM equations.

The model is tested on idealized simulations and laboratory experiments. The ability of the non-hydrostatic method to accurately represent the dispersion properties of surface waves is first demonstrated in the context of the linear theory. Hamilton and Ebersole (2001) laboratory experiment is used to evaluate the model on the issue of wave height amplification associated with beach slope as well as wave breaking. The parameterization of the wave-breaking is based on momentum horizontal diffusion, trigger conditions being related to a Froude number. The tuning of the horizontal viscosity is consistent with considerations on the mechanical energy balance. The reasoning can be extended to the possible production of turbulent kinetic energy in the turbulent closure scheme, leading in practice to the increase in vertical turbulent viscosity. If the vertical profile of the residual alongshore current can apparently be slightly improved, on the other hand, the fraction of the kinetic energy lost by the breaking wave, which can in this way be injected into the vertical 1102 turbulence, remains uncertain for the authors and requires, obviously, to be better 1103 understood. Along these lines, a part of the wave spectrum will still be missing in our future realistic applications because of the limited grid resolution (the smallest periods being concerned) with, as a result, a possible underestimation of the bottom stress in shallow areas that should be taken into account.

Finally, the Haller et al. (2002) laboratory experiment, with its bar and its two 1108 channels, makes it possible to evaluate the model on the rip current problem. The model 1109 correctly reproduces rips currents and their high temporal variability. The feedback of the rip 
1110 currents on the waves, in terms of wave amplification and refraction, is well represented. The

1111 onset of wave breaking is also well simulated, either above the bar or in the channel axis.

1112 This paper does not consider some 3D aspects as the effect of tracers on the

1113 pressure gradient and turbulence, which will be taken into account in future applications of

1114 this model. This also includes sediments that may be of great importance for modelling of rip

1115 currents as shown by $\mathrm{Ma}$ et al, 2014. These authors show that a wave-resolving non

1116 hydrostatic model is an efficient approach for the problem of resuspension of sediments by

1117 waves, and the possible feedback introduced by the modification of the equation of state by

1118 sediments, which can have a significant influence on the pressure gradient and the level of

1119 turbulence. In practice our wave model will be coupled with the MUSTANG sediment model

1120 (Le Hir et al, 2011)

1121 The next step is now to implement our model on realistic beaches, where we will

1122 confront the results with in situ data. Infragravity waves and the risk of submersion are

1123 possible applications. Some developments are still needed to provide a realistic wave

1124 spectrum to the boundary conditions of the phase-resolved wave model. The phase-

1125 averaged model, which can be applied over large domains at a reasonable cost, appears to

1126 be a good candidate for providing the boundary conditions to the phase-resolved model. The

1127 phase-averaged wave model can also provide an estimate of the missing part of the wave

1128 spectrum in the deterministic model, helping the former to improve bottom turbulence in

1129 shallow areas. Finally, wave growth by local wind action will eventually be considered in the

1130 perspective of large areas (Deigaard and Nielsen, 2018).

\section{APPENDIX A}

1133

1134 The discrete version of $\frac{\partial^{2} q}{\partial x^{2}}$ at the left-hand side of (4) is given by the left-side of:

1135

$1136 \quad \frac{q(x+d x)+q(x-d x)-2 q(x)}{d x^{2}}=\frac{\partial^{2} q}{\partial x^{2}}+\frac{d x^{2}}{12} \frac{\partial^{4} q}{\partial x^{4}}+\cdots$ 
1138 The right-hand side of $\mathrm{A} 1$ is given by a Taylor development, $\frac{d x^{2}}{12} \frac{\partial^{4} q}{\partial x^{4}}$ being the leading term of 1139 the errors made by the numerical scheme. Assuming a sinusoidal shape $\left(\frac{\partial^{4} q}{\partial x^{4}} \approx k^{4} q, k\right.$ the 1140 wave number) leads to (5).

\section{APPENDIX B: time stepping scheme}

1145 The equations are calculated with a forward-backward time stepping scheme. The 1146 alternation of the fields along the time axis is presented in Figure 19. The height of the 1147 surface at time $t, \eta^{t}$, is calculated first, knowing $\eta^{t-1}$ and velocities at time $t-1 / 2$. Equation (9) 1148 gives $q^{t}$ knowing $q^{t-1}, q^{t-2}$, and hydrostatic tendencies at time $\mathrm{t}-1$. Note that the vertical 1149 laplacian of $q$ is computed with an implicit centric scheme of the type $\frac{1}{2} \frac{\partial^{2} q^{t-2}}{\partial z^{2}}+\frac{1}{2} \frac{\partial^{2} q^{t}}{\partial z^{2}}$ so as not 1150 to limit the time step when the vertical mesh becomes very small (which becomes the case 1151 in sigma coordinates when the bathymetry tends to zero). The vertical velocity at time $\mathrm{t}^{-1} / 2$, 1152 used for the advection terms of the equations for $u$ and $v$, is computed from $u$ and $v$ at time t$11531 / 2$. Finally, the velocities at time $t+1 / 2$ are calculated from the velocities, the sea surface 1154 height and the non hydrostatic pressure of the previous times. Vertical turbulent diffusion 1155 terms are calculated with an implicit scheme.

$$
q, \eta^{t-2} \quad u, v, w^{t-3 / 2} \quad q, \eta^{t-1} \quad u, v, w^{t-1 / 2} \quad q, \eta^{t} \quad u, v, w^{t+1 / 2}
$$

Figure 19: chronology of the variables of the model. At the beginning of each iterative cycle, the 1159 fields at time t-1/2 and time tare known. 
1164 The discrete version of the left-hand side of (30) is given by the left-side of:

1165

1166

$\frac{\eta(t+d t)+\eta(t-d t)-2 \eta(t)}{d t^{2}}=\eta^{(2)}(t)+\frac{d t^{2}}{12} \eta^{(4)}(t)+\cdots$

C1

1167

1168 The right-hand side of $\mathrm{C} 1$ is given by a Taylor development, exponents in brackets indicating

1169 the order of the derivatives, $\frac{d t^{2}}{12} \eta^{(4)}(t)$ being the leading term of the errors made by the

1170 numerical scheme. Assuming a sinusoidal shape $\left(\eta^{(4)}(t) \approx \omega^{4} \eta(t), \omega\right.$ the wave frequency)

1171 leads to $\frac{d t^{2}}{12} \eta^{(4)}(t) \approx \frac{d t^{2}}{12} \omega^{4} \eta(t)$. Similar reasoning can be done on the right-hand side of

1172 (30). The leading error term of the corresponding numerical scheme is, $c^{2} \frac{d x^{2}}{12} \frac{\partial^{4} \eta}{\partial x^{4}}$, equivalent

1173 (if a sinusoidal shape is assumed, $k$ the wavenumber) to $c^{2} \frac{d x^{2}}{12} k^{4} \eta$ and $-c^{2} \frac{d x^{2}}{12} k^{2} \frac{\partial^{2} \eta}{\partial x^{2}}$. Then

1174 reformulate (30) using the first two terms of the Taylor developments leads to (31).

1175

1176

1177

\section{APPENDIX D}

1178

1179 The potential energy flux is $F=g u \eta$ with $u=g \eta / c$. A sinusoidal form $\sin \Psi$ is assumed

1180 leading to $F=g^{2} \frac{1}{c} \eta_{0}^{2} \sin ^{2} \Psi$. A wave period averaging gives $F=g^{2} \frac{1}{2 c} \eta_{0}^{2}$. Within the wave

1181 breaking zone we assume $\frac{2 \eta_{0}}{h}=\gamma$. Using $c=\sqrt{g h}$ leads to $F=\frac{1}{8} g^{3 / 2} \gamma^{2} h^{3 / 2}$. The horizontal 1182 gradient is:

1183

$1184 \quad \frac{\partial F}{\partial x}=\frac{3}{16} g^{3 / 2} \gamma^{2} h^{1 / 2} \frac{\partial h}{\partial x} \quad$ D1 
1186 Consider a constant diffusion coefficient $\left\langle v_{b}\right\rangle$. The dissipation term due to horizontal

1187 diffusion is $\varepsilon_{b}=-<v_{b}>\left(\frac{\partial u}{\partial x}\right)^{2}$. Assuming a constant wavenumber leads to $\frac{\partial u}{\partial x}=k u_{0} \cos \Psi$ 1188 and $\varepsilon_{b}=-<v_{b}>k^{2} u_{0}^{2} \cos ^{2} \Psi$. A wave period averaging gives

$1189 \varepsilon_{b}=-<v_{b}>k^{2} u_{0}^{2} \frac{1}{2}=-<v_{b}>k^{2} \frac{1}{2}\left(\frac{g \eta_{0}}{c}\right)^{2}$. Using $\frac{2 \eta_{0}}{h}=\gamma$ leads to:

1190

$1191 \quad \varepsilon_{b}=-<v_{b}>\frac{1}{8}\left(\frac{k g \gamma h}{c}\right)^{2} \quad \mathrm{D} 2$

1192

1193 Using $k=\frac{\omega}{c}=2 \pi /(c T)$ ( $T$ the wave period), the equilibrium of (D1) and (D2) leads to (35)

1194

1195

1196

1197

1198

1199

Acknowledgements

1200

1201

The authors acknowledge the support of the SIROCCO team http://sirocco.obs-mip.fr/, the

1202

NUMEROFIX project (NUmerical ModElling of Real Ocean surFace mIXing) funded by

1203 LEFE/GMMC, the HPC resources from CALMIP (Grant 2018-P1325) and from GENCI and

1204 CINES (Grand Equipement National de Calcul Intensif, project A0040110088).

1205

1206

1207

References

1208

1209 Ardhuin, F., Rascle, N., Belibassakis, K., 2008. Explicit wave-averaged primitive equations

1210 using a generalized Lagrangian mean, Ocean Modelling, 20, 35-60, 1211 doi:10.1016/j.ocemod.2007.07.001. 
1213 Benetazzo A., Carniel S., Sclavo M., A. Bergamasco A., 2013. Wave-current interaction:

1214 Effect on the wave field in a semi-enclosed basin. Ocean Modelling 70, 152-165.

1215 http://dx.doi.org/10.1016/j.ocemod.2012.12.009

1216

1217 Bennis, A., Ardhuin, F., Dumas, F., 2011. On the coupling of wave and three-dimensional 1218 circulation models: Choice of theoretical framework, practical implementation and adiabatic 1219 tests, Ocean Model., 40, 260-272.

1220

1221

1222

1223

Benoit, M., Raoult C., Yates M.L., 2017. .Analysis of the linear version of a highly dispersive potential water wave model using a spectral approach in the vertical. Wave Motion 74,159 181.doi:10.1016/j.wavemoti.2017.07.002

1224

1225

Berkhoff J., 1972. Computation of combined refraction-diffraction, in: Proc. 13th Int. Conf. on 1226 Coastal Eng., vol. 1, pp. 471-490.

1227

1228

Bertin, X., Bruneau, N., Breilh, J.-F., Fortunato, A. B., Karpytchev M., Importance of wave 1229 age and resonance in storm surges, The case Xynthia, Bay of Biscay, 2012. Ocean 1230 Modelling, Volume 42, $\quad$ Pages 16-30, ISSN 1463-5003, https://doi.org/10.1016/j.ocemod.2011.11.001.

1233 Bertin, X., de Bakker, A., van Dongeren, A., Coco, G., André, G., Ardhuin, F., Bonneton, P., 1234 Bouchette, F., Castelle, B., Crawford, W. C., Davidson, M., Deen, M., Dodet, G., Guérin, T., 1235 Inch, K., Leckler, F., McCall, R., Muller, H., Olabarrieta, M., Roelvink, D., Ruessink, G., 1236 Sous, D. , Stutzmann, E., Tissier, M., 2018. Infragravity waves: From driving mechanisms to 1237 impacts. $\quad$ Earth-Science $\quad$ Reviews $\quad 177 \quad$ 774-799. 1238 https://doi.org/10.1016/j.earscirev.2018.01.002 
1240 Blayo, E., Debreu, L., 2005. Revisiting open boundary conditions from the point of view of 1241 characteristic variables. Ocean Modelling 9, 231-252.

1242

1243 Blumberg, A.F., Mellor, G.L., 1987. A description of a three-dimensional coastal circulation 1244 model. In: Heaps, N. (Ed.), Three-Dimensional Coastal Ocean Models, Coastal Estuarine 1245 Science, vol. 4. American Geophysical Union, pp. 1-16.

1246

1247 Bonneton, P., Chazel, F., Lannes, D., Marche, F., Tissier, M., 2011a. J. Comput. Phys., 230, 1248 1479-1498, DOI:10.1016/j.jcp.2010.11.015

1249

1250 Bonneton, P., Barthelemy, E., Chazel, F., Cienfuegos, R., Lannes, D., Marche, F., Tissier, 1251 M., 2011b. Recent advances in Serre-Green Naghdi modelling for wave transformation, 1252 breaking and runup processes, 2011b. European Journal of Mechanics - B/Fluids, 30, 5891253 597, https://doi.org/10.1016/j.euromechflu.2011.02.005.

1254

1255 Bouharguane A., Azerad P., Bouchette F., Marche F., Mohammadi B., 2010. Low complexity 1256 shape optimization \& a posteriori high fidelity validation. Discrete \& Continuous Dynamical 1257 Systems - B, 13 (4) : 759-772. doi: $\underline{10.3934 / d c d s b .2010 .13 .759}$

1258

1259 Bordois, L., Auclair, F., Paci, A., Dossmann, Y., and Nguyen, C., 2017. Nonlinear processes 1260 generated by supercritical tidal flow in shallow straits. Physics of Fluids, $1261 \quad$ http://dx.doi.org/10.1063/1.4986260

1262

1263 Bruneau, N., 2009. Modélisation morphodynamique des plages sableuses,Ph.D. thesis, 1264 Université Bordeaux I. 
1266 Castelle, B., Scott, T., Brander, R.W., McCarroll R.J., 2016. Rip current types, circulation 1267 and hazard, In Earth-Science Reviews, Volume 163, Pages 1-21, ISSN 0012-8252, 1268 https://doi.org/10.1016/j.earscirev.2016.09.008.

1269

1270 Costa, A., Doglioli, A. M., Marsaleix, P., Petrenko A. A., 2017. Comparison of in situ 1271 microstructure measurements to different turbulence closure schemes in a 3-D numerical 1272 ocean circulation model. Ocean Modelling. https://doi.org/10.1016/..ocemod.2017.10.002

1273

1274 Deigaard, R. and Nielsen, P., 2018. Wind generation of waves: Energy and momentum 1275 transfer - An overview with physical discussion, Coastal Engineering, 139, 36-46, 1276 https://doi.org/10.1016/j.coastaleng.2018.04.024.

1277

1278 Feddersen, F., Trowbridge, J.H., 2005. The Effect of Wave Breaking on Surf-Zone 1279 Turbulence and Alongshore Currents: A Modelling Study. Journal of physical oceanography. $1280 \quad 2187-2203$

1281

1282 Feddersen, F., Trowbridge, J.H., Williams III, A.J., 2007. Vertical Structure of Dissipation in 1283 the Nearshore. Journal of Physical Oceanography. 37, 1764-1777. 1284 http://dx.doi.org/10.1175/JPO3098.1

1285

1286 Guérin, T., Bertin, X., Coulombier, T., de Bakker, A., 2018. Impacts of wave-induced 1287 circulation in the surf zone on wave setup, Ocean Modelling, 123, 86-97, 1288 https://doi.org/10.1016/i.ocemod.2018.01.006.

1290 Green, A.E., Naghdi, P.M., 1976. A derivation of equations for wave propagation in water of 1291 variable depth. J. Fluid Mech., 78, 237-246 
1293 Haller, M. C., Dalrymple, R. A., Svendsen, I. A., 2002. Experimental study of nearshore 1294 dynamics on a barred beach with rip channels, J. Geophys. Res., 107(C6), doi: $1295 \quad 10.1029 / 2001 J C 000955$.

1296

1297 Hamilton, D. G., and B. A. Ebersole, 2001. Establishing uniform longshore currents in a 1298 large-scale sediment transport facility, Coastal Engineering, 42 , 199-218.

1299

1300 Haas, K. A., and I. A. Svendsen, 2002. Laboratory measurements of the vertical structure of 1301 rip currents, J. Geophys. Res., 107(C5), doi: 10.1029/2001JC000911.

1302

1303 Haas, K. A., Svendsen, I. A., Haller, M. C. Zhao, Q. 2003. Quasi $\square$ three $\square$ dimensional 1304 modelling of rip current systems, J. Geophys. Res., 108, 3217, doi: 10.1029/2002JC001355, 1305 C7.

1306

1307

Haas, K. A., Warner, J. C., 2009. Comparing a quasi-3D to a full 3D nearshore circulation 1308 model: SHORECIRC and ROMS, Ocean Modelling, Volume 26, Issues 1-2, 91-103, https://doi.org/10.1016/j.ocemod.2008.09.003.

1310

1311 Kanarska, Y., Maderich, V., 2003. A non-hydrostatic numerical model for calculating free1312 surface stratified flows. Ocean Dyn. 53 (3), 176-185.

1313

1314 Kanarska, Y., Shchepetkin, A., McWilliams, J. C., 2007. Algorithm for non-hydrostatic 1315 dynamics in the Regional Oceanic Modelling System, In Ocean Modelling, Volume 18, 1316 Issues 3-4, 143-174, https://doi.org/10.1016/j.ocemod.2007.04.001.

1318 Kennedy, A. B., Chen, Q., Kirby, J. T., Dalrymple, R. A. 2000. Boussinesq modelling of wave 1319 transformation, breaking and runup. I: 1D. J. Waterw., Port, Coastal, Ocean Eng., 1261, 39132047. 
1322 Kinsman, B., 1965. Wind waves: Their Generation and Propagation on the Ocean Sur-

1323 face. Courier Dover Publications.

1324

1325 Kirby, J.T. and Dalrymple, R.A., 1983. A parabolic equation for the combined refraction1326 diffraction of Stokes waves by mildly-varying topography, Journal of Fluid Mechanics, 136, $1327 \quad 453-466$.

1328

1329 Kirby, J.T., Wei, G., Chen, Q., Kennedy, A., Dalrymple, R.A., 1998. FUNWAVE 1.0; Fully 1330 Nonlinear Boussinesq Wave Model Documentation and User's Manual. Newark, Delaware: 1331 Center for Applied CoastResearch, University of Delaware, Research Report CACR-98-06.

1332

1333 Klingbeil, K., Burchard, H., 2013. Implementation of a direct nonhydrostatic pressure 1334 gradient discretisation into a layered ocean model, Ocean Modelling, 65, 64-77, 1335 https://doi.org/10.1016/j.ocemod.2013.02.002

1336

1337 Klingbeil, K., Debreu, L., Lemarié, F., Burchard, H., 2018. The numerics of hydrostatic 1338 structured-grid coastal ocean models: state of the art and future perspectives. Ocean 1339 Modelling.

1341 Kumar, N., Feddersen, F., Uchiyama, Y., McWilliams, J., O’Reilly, W, 2015. Midshelf to 1342 Surfzone Coupled ROMS-SWAN Model Data Comparison of Waves, Currents, and 1343 Temperature: Diagnosis of Subtidal Forcings and Response. J. Phys. Oceanogr., Vol. 45, 1344 pp. 1464-1490, DOI: 10.1175/JPO-D-14-0151.1

1346 Lee, J.W., Teubner, M.D., Nixon, J.B., Gill, P.M., 2006. Application of the artificial 1347 compressibility method for turbulent open channel flows. International Journal for Numerical 1348 Methods in Fluids 51, 617-633. 
1350 Le Hir P., Cayocca F., Waeles B., 2011. Dynamics of sand and mud mixtures: A 1351 multiprocess-based modelling strategy. Continental Shelf Research, 31, 135-149.

1352 https://doi.org/10.1016/j.csr.2010.12.009

1353

1354 Lemarié, F., Debreu, L., Madec, G., Demange, J., Molines, J.M., Honnorat, M., 2015.

1355 Stability constraints for oceanic numerical models: implications for the formulation of time 1356 and space discretizations, Ocean Modelling, 92, 124-148, 1357 https://doi.org/10.1016/j.ocemod.2015.06.006.

1358

1359

1360

Lynett, P., 2006: Nearshore wave modelling with high-order Boussinesq-type equations. J Waterw Port C-ASCE, 132, 348-357, doi:10.1061/(ASCE)0733-950X(2006)132:5(348).

1361

1362

Mahadevan, A., Oliger, J., Street, R., 1996. A nonhydrostatic mesoscale ocean model parts 1363 1, 2, J. Phys. Oceanogr. 26 (9), 1868-1900.

1364

1365

Marshall, J., Hill, C., Perelman, L., Adcroft, A., 1997. Hydrostatic, quasi-hydrostatic, and 1366 nonhydrostatic ocean modelling. J. Geophys. Res. 102, 5733-5752.

1367

1368

Ma, G., Shi, F., Kirby, J. T., 2012. Shock-capturing non-hydrostatic model for fully dispersive surface wave processes.

Ocean Modelling, 43-44, 22-35,

1370 https://doi.org/10.1016/j.ocemod.2011.12.002.

1372 Ma, G., Chou, Y.-J., Shi, F., 2014. A wave-resolving model for nearshore suspended 1373 sediment transport. Ocean Modelling, 77 , 33-49,

1374 https://doi.org/10.1016/j.ocemod.2014.03.003. 
1376 Magne, R., Ardhuin, F., Rey, V., Herbers, T. H. C., 2005. Topographical Scattering of

1377 Waves: Spectral Approach. Journal of Waterway, Port, Coastal \& Ocean Engineering. Vol. 1378131 Issue 6, p311-320. 10p. 15 Charts. DOI: 10.1061/(ASCE)0733-950X(2005)131:6(311)

1379

1380 Marsaleix P., Auclair F., Estournel C., 2006, Considerations on Open Boundary Conditions 1381 for Regional and Coastal Ocean Models. Journal of Atmospheric and Oceanic Technology, 1382 23,1604-1613, http://dx.doi.org/10.1175/JTECH1930.1

1383

1384 Marsaleix, P., Auclair, F., Floor, J. W., Herrmann, M. J., Estournel, C., Pairaud, I., Ulses, C., 1385 2008. Energy conservation issues in sigma-coordinate free-surface ocean models. Ocean 1386 Modelling. 20, 61-89. http://dx.doi.org/10.1016/j.ocemod.2007.07.005

1387

1388 Marsaleix, P., Auclair, F., Duhaut, T., Estournel, C., Nguyen, C., Ulses, C., 2012. 1389 Alternatives to the Robert-Asselin filter. Ocean Modelling, 41, 53-66 1390 http://dx.doi.org/10.1016/j.ocemod.2011.11.002

1391

1392

McKiver, W. J., Sannino, G., Braga, F., Bellafiore, D., 2016. Investigation of model capability 1393 in capturing vertical hydrodynamic coastal processes: a case study in the north Adriatic Sea. Ocean Science, 12, 51-69, http://dx.doi.org/10.5194/os-12-51-2016

1395

McWilliams, J. C., Restrepo, J. M., Lane, E. M., 2004. An asymptotic theory for the 1397 interaction of waves and currents in coastal waters, J. Fluid Mech., 511, $135-178$.

Mellor, G., 2003. The Three-Dimensional Current and Surface Wave Equations. Journal of 1400 Physical Oceanography, Vol. 33 Issue 9, p1978. 12p. 
1402 Michaud, H., Marsaleix, P., Leredde, Y., Estournel, C., Bourrin, F., Lyard, F., Mayet, C.,

1403 Ardhuin, F., 2012. Three-dimensional modelling of wave-induced current from the surf zone

1404 to the inner shelf. Ocean Science, 8, 657-681. http://dx.doi.org/10.5194/os-8-657-2012

1405

1406 Miche, A., 1944: Mouvements ondulatoires de la mer en profondeur croissante ou

1407 décroissante. forme limite de la houle lors de son déferlement. application aux digues

1408 maritimes. Troisième partie. Forme et propriétés des houles limites lors du déferlement.

1409 Croissance des vitesses vers la rive. Annales des Ponts et Chaussées, Tome 114, 369-406.

1410

1411 Oger, G., 2006. Aspects théoriques de la méthode SPH et applications à l'hydrodynamique 1412 à surface libre, PhD Thesis, Ecole Centrale de Nantes, France.

1413

1414 Paluszkiewicz, T., Garwood, R.W., Denbo, D.W., 1994. Deep convective plumes in the 1415 ocean. Oceanography 7(2):37-44, http://dx.doi.org/10.5670/oceanog.1994.01.

1416

1417 Petrenko A., Dufau C., Estournel C., 2008, Barotropic eastward currents in the western Gulf 1418 of Lion, north-western Mediterranean Sea, during stratified conditions. Journal of Marine 1419 Systems, 74, 406-428, doi:10.1016/j.jmarsys.2008.03.004

1421 Raoult, C., Benoit, M., Yates, M. L., 2016. Validation of a fully nonlinear and dispersive wave 1422 model with laboratory non-breaking experiments. Coastal Engineering, 114, 194-207 1423 doi:10.1016/j.coastaleng.2016.04.003.

1425 Rodi, W., 1987. Examples of calculation methods for flow and mixing in stratified fluid. J. 1426 Geophys. Res., 92, 5305-5328.

1428 Roeber, V. and Cheung, K. F., 2012. Boussinesq-type model for energetic breaking waves in 1429 fringing reef environments. Coastal Engineering, 70:1-20. 
1431 Roullet, G., Molemaker, M. J., Ducousso, N., Dubos, T., 2017. Compact symmetric Poisson 1432 equation discretization for non-hydrostatic sigma coordinates ocean model, Ocean 1433 Modelling, 118, 107-117, https://doi.org/10.1016/j.ocemod.2017.09.001.

1435 Rusu, E., Soares, C. G., 2013. Modelling Waves in Open Coastal Areas and Harbors with 1436 Phase-Resolving and Phase-Averaged Models. Journal of Coastal Research, Vol. 29, No. 6. 1437 DOI: 10.21121JCOASTRES-D-11-00209.1

1438

1439 Serre, F., 1953. Contribution à l'étude des écoulements permanents et variables dans les 1440 canaux. La Houille Blanche, 3 (6), 374-388

1441

1442 Shi, J., Shi, F., Kirby, J. T., Ma, G., Wu, G., Tong, C., Zheng J., 2015. Pressure Decimation 1443 and Interpolation (PDI) method for a baroclinic non-hydrostatic model, In Ocean Modelling, 1444 Volume 96, Part 2, Pages 265-279, ISSN 1463-5003, 1445 https://doi.org/10.1016/.ocemod.2015.09.010.

1447 Soulsby, R., 1995. Bed shear stresses due to combined waves and currents. In: Stive, M., 1448 Fredsøe, J., Hamm, L., Soulsby, R., Teisson, C., Winterwerp, J. (Eds.), Advances in Coastal 1449 Morphodynamics. Delft Hydraulics, Delft, The Netherlands, pp. 420-423.

1451 Svendsen, I. A., Qin, W., Ebersole, B. A., 2003. Modelling waves and currents at the LSTF 1452 and other laboratory facilities. Coastal Engineering 50, $19 \quad$ - 45. $1453 \quad$ http://dx.doi.org/10.1016/S0378-3839(03)00077-2

1455 Tissier, M., Bonneton, P., Marche, F., Chaze, I F., Lannes, D., 2012. A new approach to 1456 handle wave breaking in fully non-linear Boussinesq models, Coastal Engineering, 67, 541457 66, https://doi.org/10.1016/j.coastaleng.2012.04.004. 
1459 Tolman, $\mathrm{H}$. et al. 2016. User manual and system documentation of WAVEWATCH III (R)

1460 version 5.16.NOAA/NWS/NCEP/MMAB Tech. Note. 329. 326 pp. +Appendices

1461

1462 Uchiyama, Y., McWilliams, J. C., Restrepo, J. M., 2009, Wave $\square$ current interaction in 1463 nearshore shear instability analyzed with a vortex force formalism, J. Geophys. Res., 114, 1464 C06021, doi:10.1029/2008JC005135.

1465

1466 Uchiyama, Y., McWilliams, J. C., Shchepetkin, A. F., 2010. Wave-current interaction in an 1467 oceanic circulation model with a vortex-force formalism: Application to the surf zone, Ocean 1468 Model., 34, 16-35, doi:10.1016/j.ocemod.2010.04.002.

1469

1470 Uchiyama, Y., J. C. McWilliams, Akan, C., 2017. Three-dimensional transient rip currents: 1471 Bathymetric excitation of low-frequency intrinsic variability, J. Geophys. Res. Oceans, 122, 1472 5826-5849, doi:10.1002/2017JC013005.

1473

1474 Weir, B., Uchiyama, Y., Lane, E. M., Restrepo, J. M., Williams,J. M., 2011. A vortex force 1475 analysis of the interaction of rip currents and surface gravity waves, J. Geophys. Res., 116, 1476 C05001, doi:10.1029/2010JC006232.

1478 Yoon, J. J., 2014. Non-hydrostatic Modelling of Waves Transformation and Rip Current 1479 circulation: A case Study for Haeundae Beach, Korea. Journal of Coastal Research, 72, 1841480189.

1482 Zijlema, M., Stelling, G., Smit, P., 2011. SWASH: An operational public domain code for 1483 simulating wave fields and rapidly varied flows in coastal waters. Coastal Engineering, 58, 1484 992-1012, https://doi.org/10.1016/j.coastaleng.2011.05.015. 
1486 Zhou, Z., Hsu, T.-J., Cox, D., Liu, X., 2017. Large $\square$ eddy simulation of wave $\square$ breaking

1487 induced turbulent coherent structures and suspended sediment transport on a barred beach.

1488 Journal of Geophysical Research: Oceans 122 (1), 207-235. doi: 10.1002/2016JC011884 